\title{
Multi-Plet Two-Channel Perfect Reconstruction FIR and Causal Stable IIR Filter Banks
}

\author{
K. M. Tsui and S. C. Chan, Member, IEEE
}

\begin{abstract}
This paper proposes a new class of two-channel structural perfect reconstruction (PR) finite-impulse response / infiniteimpulse response (FIR/IIR) filter banks (FBs) called the multi-plet FBs. It generalizes the structural PR FBs proposed by Phoong $\mathrm{et} \mathrm{al}$. and the conventional triplet FB in that it employs multiple lifting steps similar to the conventional lifting structure. Apart from the important structural PR property, the multi-plet FB can be systematically designed to meet a given specification on the passband/ stopband ripples and transition bandwidth: a low-order prototype PR FB with a much wider transition band is first designed using nonlinear optimization in order to obtain a symmetric frequency response and prescribed passband and stopband ripples. As only a few variables are required, its performances can easily be controlled. A subfilter is then designed using second-order cone programming (SOCP) so that the prototype FB can be warped by means of frequency transformation to meet the desired transition bandwidth, while preserving the PR condition, passband/stopband ripples and lifting structure of the prototype FB. The design procedure is very general and it applies to both linear-phase/low-delay FIR and IIR multi-plet FBs. By formulating the $K$-regularity conditions as a set of linear equality constraints on the subfilter coefficients, multi-plet-based wavelet bases can easily be designed under the SOCP framework. Design examples show that the proposed approach offers more flexibility in controlling the frequency characteristics of the PR FBs and lower design complexity than conventional methods.
\end{abstract}

Index Terms-Causal stable infinite-impulse response (IIR), finite-impulse response (FIR), $\boldsymbol{K}$-regularity, lifting structure, lowdelay, multi-plet, perfect reconstruction (PR) filter banks (FBs), second-order cone programming (SOCP), structural PR, wavelet bases.

\section{INTRODUCTION}

$\mathbf{P}$ ERFECT reconstruction (PR) filter banks (FBs) have important applications in signal analysis, coding, and the design of wavelet bases. An efficient structure of two-channel finite-impulse response (FIR)/allpass FBs, which structurally satisfies the PR condition, was the structural PR FB proposed by Phoong et al. [1]. Subsequent works for the design of low-delay FIR and infinite-impulse response (IIR) structural PR FBs and wavelet bases can be found in [2]-[6]. One limitation of this structure is that the magnitudes of the low-pass and high-pass analysis filters at $\omega=\pi / 2$ in the linear-phase case are respectively restricted to 0.5 and 1 , or vice versa, which make the frequency response of the FB asymmetric. In another structural PR FBs called triplet FBs [7], a generalization of the structure in [1],

Manuscript received April 19, 2005; revised September 14, 2005 and April 28, 2006. This paper was recommended by Associate Editor V. E. DeBrunner.

The authors are with the Department of Electrical and Electronic Engineering, The University of Hong Kong, Hong Kong (e-mail: kmtsui@eee.hku.hk; scchan@eee.hku.hk).

Digital Object Identifier 10.1109/TCSI.2006.885694 more symmetric frequency responses can be obtained by properly choosing the parameters in the structure. The triplet FBs are parameterized by three subfilters and three delay parameters. To meet different design specifications, these subfilters can be chosen as arbitrary functions such as linear-phase FIR [7]-[9] and low-delay FIR [10], [11] functions. Because of the structural PR property, the design of PR FBs can be simplified to general filter design problems.

The flexibility and effectiveness of the abovementioned structural PR FBs motivate us to study further a new class of two-channel structural PR FBs with multiple lifting steps called the multi-plet FBs. The proposed approach can be regarded as an extension of the structural PR FBs [1]-[6] and the triplet FBs [7]-[11], which are based on the lifting structure with two and three lifting steps [12], [13], respectively. Furthermore, it is shown later in this paper that the design of the proposed multi-plet FB is closely related to the concept of frequency transformation of digital filters studied by Saramaki [14] and Kaiser et al. [15]. As a result, the frequency characteristics of the multi-plet PR FB can be varied online by varying the subfilter or the transformation kernel. More precisely, if an two-channel PR FB is expressed in certain ladder or lifting structure having the same subfilter of the form $\left(1+z^{-1}\right) / 2$ in all the lifting steps, then appropriate frequency transformation similar to the approach in [14] and [15] can be applied directly to the lifting structure to obtain another PR FB with the same number of lifting steps having the same passband and stopband ripples but an arbitrary sharp transition bandwidth. Thus, the design of the multi-plet FB can be divided into two sub-problems: i) the design of a prototype PR FB to meet a certain specification on the passband and stopband ripples; and ii) the design of a subfilter which determines the cutoff frequency of the final multi-plet FB after transformation.

The proposed multi-plet FB is particularly useful in designing two-channel PR FB with narrow transition bandwidth. To design such narrow transition band FBs, a prototype PR FB with a much wider transition bandwidth is first designed using nonlinear optimization in order to obtain a symmetric response and given passband and stopband ripples. As only a few variables are required, its performances can easily be controlled. By properly designing the subfilter, the frequency response of the prototype FB can be warped by means of frequency transformation to meet the desired transition bandwidth, while preserving the PR condition. The transformed multi-plet FB can also be implemented as a lifting structure with the same number of lifting steps as its prototype. Like the structural PR and triplet FBs, the PR condition is preserved under coefficient quantization. Another important advantage of this structure is that the lengths and the passband delays 
of the analysis/synthesis filters can be made closer to each other, which simplifies practical implementation and reduces the number of additional delay elements required to process the subband signals. Moreover, a systematic design procedure and design formulae for the proposed method are available to meet a given specification on the passband and stopband ripples and transition bandwidth of the final PR FB.

The major difficulty in step i) of the design of multiplet PR FB is the determination of the lifting coefficients in the lifting structure. For the conventional triplet FBs [7] with three lifting steps, the prototype PR FB has relatively fewer degree of freedom than the multi-plet FB and the lifting coefficients can be determined by considering the responses at certain frequency points, say $\omega=0, \pi / 2$ and $\pi$. In the multi-plet case, the determination of these coefficients becomes a nontrivial task since the lifting factorization using Euclidean factorization [12], [13] is in general nonunique and there are many possible choices of these coefficients. To overcome this problem, a prototype PR FB with type 1 FIR linear-phase analysis filters (i.e filters with odd length and symmetric impulse response) is employed so that it can be factored uniquely into a ladder or lifting structure [16], [17] and subsequently transformed to the meet the desired specifications using the techniques in [14] and [15].

Apart from designing linear-phase PR FBs, we found that the proposed design approach is also applicable to the design of low-delay multi-plet FBs. For FIR linear-phase subfilters, the transformed multi-plet FB is a FIR linear-phase FB and its system delay is governed by the length of the subfilter and the number of lifting steps (i.e., the length of the prototype FB). To reduce the system delay, approximately linear-phase FIR subfilters can be used instead of linear-phase FIR subfilters as in [2], [4], [10] and [11]. Design results show that the proposed low-delay multi-plet FB offers a comparable performance as, but considerably lower system delay than, their linear-phase counterparts. In addition, we also extend this method to the design of causal stable IIR subfilters using the model reduction technique in [18] and [19]. The idea is to determine initially the denominator of the IIR function given the target FIR subfilter, and then optimally design the numerator using second-order cone programming (SOCP) given the denominator determined at the first stage. This approach has the advantages of simple design procedure and the ability to preserve properties of the target FIR subfilter. Moreover, the stability of the IIR subfilter so obtained is guaranteed. Additional constraints, such as prescribed $K$-regularity or peak ripple constraints, can be readily imposed to improve the frequency characteristics of the resulting IIR multi-plet FB. Consequently, causal stable IIR multi-plet FBs and wavelet bases can be obtained. Similar to the works in [1] and [20], the proposed multi-plet FB can also be extended to multi-dimensional FBs [21].

For the design of the FIR/IIR subfilters in step ii) above, we formulate the design problem as a convex programming problem and solve it using SOCP [22]-[25]. Alternatively, another flexible convex optimization tool called semidefinite programming (SDP) [4], [10], [11], [26] can also be used. The main advantage of using SOCP and SDP is that the problem is convex and the global optimal solution, if it exists, is guaranteed. Furthermore, a wide variety of constraints such as

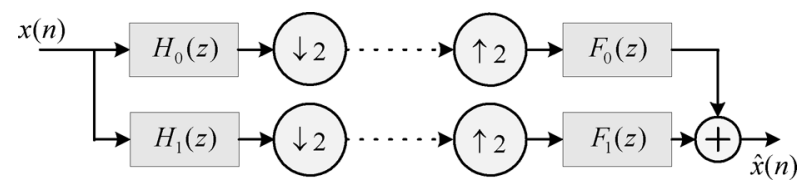

Fig. 1. Two-channel maximally decimated FB.

linear equality and inequality constraints and convex quadratic inequality constraints can be imposed readily to meet different design objectives and constraints. Interested readers are referred to [22]-[25] for more details of SOCP and its application to filter design problems. Using the SOCP formulation, it is also possible to impose prescribed $K$-regularity constraints on the multi-plet FBs in order to obtain muti-plet-based wavelet bases. The required $K$-regularity condition is a set of linear equality constraints on the subfilter. Interestingly enough, the transformed multi-plet FBs can be used to construct wavelet bases by an appropriate transformation, even though the prototype FB is not a wavelet FB.

It should be noted that there are previous works on the design of two-channel PR FBs using frequency transformation [6], [20], [27]. The present work extends the frequency transformation concept in [14] and [15] to the lifting structure, which was also pointed out in the conclusion of $[28$, section $\mathrm{V}, 2 \mathrm{nd}$ paragraph]. In the proposed frequency transformation approach, it is possible to derive a systematic procedure and design formulae to meet a given design specifications of passband and stopband ripples and transition bandwidth. These relations between the prototype FB and the transformed FB were not discussed in detail in [6], [20] and [27] so that it is not clear how to control the frequency characteristics of the latter precisely. In addition, we also study low-delay FIR PR FBs in this work, while [20] and [27] mainly focus on linear-phase FIR PR FBs. The paper is organized as follows: the proposed multi-plet FBs and the concept of frequency transformation are introduced in Section II. Section III is devoted to the design of the prototype FBs and the factorization of the prototype FBs into multiple lifting steps. The FIR/IIR subfilter design problems are addressed in Sections IV and V, where the proposed SOCP formulations are given. The derivation of the prescribed $K$-regularity constraints and a method for imposing these constraints will also be introduced. The effectiveness of the proposed approach is then demonstrated by several design examples in Section VI, and finally, conclusion is drawn in Section VII.

\section{Multi-Plet Two-Channel PR FIR FBs}

Fig. 1 shows the general structure of two-channel maximally decimated FB. The $z$-transform of the output is

$$
\begin{aligned}
\hat{X}(z)=\left[H_{0}(z) F_{0}(z)+H_{1}(z) F_{1}(z)\right] X(z) & \\
+ & {\left[H_{0}(-z) F_{0}(z)+H_{1}(-z) F_{1}(z)\right] X(-z) . }
\end{aligned}
$$

The two-channel FB is PR if $H_{0}(z) F_{0}(z)+H_{1}(z) F_{1}(z)=$ $c z^{-n_{d}}$ and $H_{0}(-z) F_{0}(z)+H_{1}(-z) F_{1}(z)=0$, where $n_{d}$ is an integer and $c$ is a nonzero constant. Therefore, the input and output of the PR FB are identical up to a time delay of $n_{d}$ samples and scaling, i.e., $\hat{x}(n)=c x\left(n-n_{d}\right)$. 


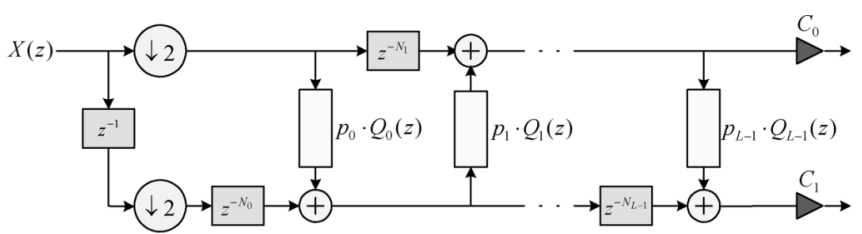

(a)

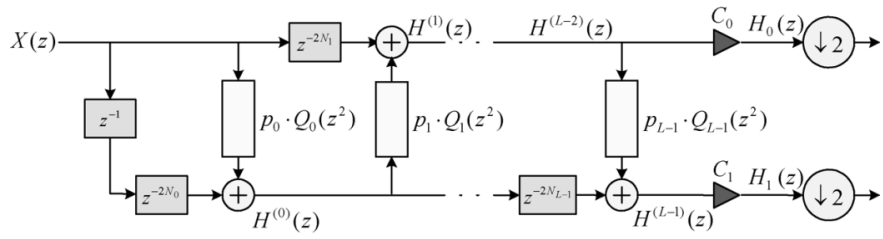

(b)
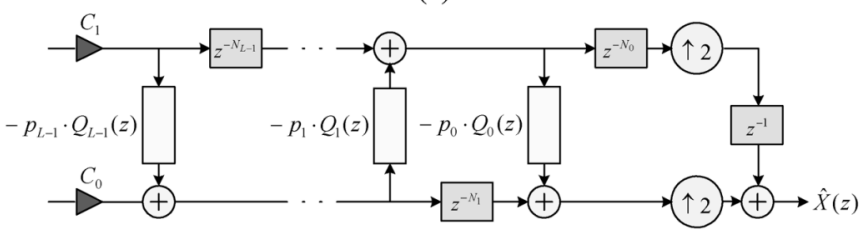

(c)

Fig. 2. General structure of the two-channel multi-plet FB. Analysis bank: (a) before and (b) after the application of noble identity. (c) Synthesis bank.

\section{A. Lifting Structure}

The general structure of the multi-plet two-channel analysis FBs is shown in Fig. 2(a). It is parameterized by $L$ subfilters $Q_{m}(z), L$ delay parameters $N_{m}, L$ lifting coefficients $p_{m}$, and 2 scaling constants $C_{0}$ and $C_{1}$ for $m=0,1, \ldots, L-1$. For convenience, let us consider Fig. 2(b), which is obtained from Fig. 2(a) by moving the decimators to the right of the subfilters using the noble identity [29]. The $z$-transform of the intermediate filter $H^{(m)}(z)$ after the $m$ th lifting step can be written as the following recursion:

$$
\begin{aligned}
H^{(0)}(z) & =z^{-2 N_{0}-1}+p_{0} \cdot Q_{0}\left(z^{2}\right) \\
H^{(1)}(z) & =z^{-2 N_{1}}+p_{1} \cdot Q_{1}\left(z^{2}\right) H^{(0)}(z) \\
H^{(m)}(z) & =z^{-2 N_{m}} H^{(m-2)}(z)+p_{m} \cdot Q_{m}\left(z^{2}\right) H^{(m-1)}(z)
\end{aligned}
$$

for $m=2,3, \ldots, L-1$. For reasons to be apparent later (please see Section II-B), we assume that the lengths of $H_{0}(z)$ and $H_{1}(z)$, denoted respectively by $L_{0}$ and $L_{1}$, are odd integers with $L_{1}>L_{0}$. Hence, the $z$-transforms of the resultant analysis and synthesis filters in the lifting structure can be written as follows:

$$
\begin{aligned}
H_{0}(z) & =C_{0} H^{(L-2)}(z) \text { and } H_{1}(z)=C_{1} H^{(L-1)}(z) \\
F_{0}(z) & =-H_{1}(-z) \text { and } F_{1}(z)=H_{0}(-z) .
\end{aligned}
$$

It can be verified that the synthesis filters can be realized using the structure in Fig. 2(c). Next, we shall consider a special case of lifting with identical subfilters

$$
Q_{L-1}\left(z^{2}\right)=\cdots=Q_{1}\left(z^{2}\right)=Q_{0}\left(z^{2}\right)=Q\left(z^{2}\right) .
$$

For causal implementation of such FB, the delay parameters $N_{m}$ should be chosen as follows:

$$
N_{L-1}=\cdots=N_{2}=N_{1}=G \text { and } N_{0}=\frac{(G-1)}{2}
$$

where $G$ is the passband group delay of $Q\left(z^{2}\right)$. As a result, the group delays of the analysis filter pair, $H_{0}(z)$ and $H_{1}(z)$, are, respectively, given by

$$
G_{0}=(L-1) \cdot G \text { and } G_{1}=L \cdot G .
$$

It will be seen later in Section III that an odd-length linear-phase PR FB can always be factored in such a form, called lifting structure, using Euclidean factorization.

\section{B. Transformation of the Lifting Structure}

If the lifting coefficients are properly chosen, multi-plet FBs with good frequency characteristic can be obtained and they are structural PR for arbitrary choice of the subfilters, which includes linear-phase/low-delay FIR and IIR filters. However, the determination of these coefficients is a nontrivial task since the lifting factorization using Euclidean factorization [12], [13] is in general nonunique and there are many possible choices of these coefficients. To overcome this problem, a prototype PR FB with type 1 FIR linear-phase analysis filters (i.e., filters with odd length and symmetric impulse response) is employed. Furthermore, assume that the lengths of the analysis filters differ by two, then the prototype PR FB can be factored uniquely into a ladder or lifting structure having the same subfilter of the form $\left(1+z^{-1}\right) / 2$ in all the lifting steps. In the proposed approach, the variable $x=\left(z+z^{-1}\right) / 2$, which is derived from the above term $\left(1+z^{-1}\right) / 2$ in the lifting step, will be transformed to obtain a new FB. To avoid any confusion, all the symbols associated with the prototype FB before transformation are augmented by the symbol " ". For instance, the $z$-transform variable $z$ and its associated variable $x$ as mentioned above for the prototype FB are now denoted by $\widetilde{z}$ and $\widetilde{x}$, respectively. Similarly, the various $z$-transforms of the prototype filters $H_{i}(z), H^{(m)}(z), Q_{m}(z)$ and $Q(z)$ are now respectively replaced by $\widetilde{H}_{i}(\widetilde{z}), \widetilde{H}^{(m)}(\widetilde{z})$, $\widetilde{Q}_{m}(\widetilde{z})$ and $\widetilde{Q}(\widetilde{z})$. For filters and FBs after transformation, and other common parameters such as $N_{m}, p_{m}$ and $C_{\boldsymbol{i}}$, the conventional notations without the symbol " $\sim$ " will be employed.

Let the zero-phase responses of $\widetilde{H}^{(m)}(\widetilde{z})$, denoted by $\widetilde{R}^{(m)}(\widetilde{x})$, for $m=0,1, \ldots, L-1$ be given by

$$
\begin{aligned}
\widetilde{R}^{(0)}(\widetilde{x}) & =\widetilde{z} \widetilde{H}^{(0)}(\widetilde{z})=1+p_{0} \widetilde{x} \\
\widetilde{R}^{(1)}(\widetilde{x}) & =\widetilde{z}^{2} \widetilde{H}^{(1)}(\widetilde{z})=1+p_{1} \widetilde{x} \cdot \widetilde{R}^{(0)}(\widetilde{x}) \\
\widetilde{R}^{(m)}(\widetilde{x}) & =\widetilde{z}^{m+1} \widetilde{H}^{(m)}(\widetilde{z})=\widetilde{R}^{(m-2)}(\widetilde{x})+p_{m} \widetilde{x} \cdot \widetilde{R}^{(m-1)}(\widetilde{x}) .
\end{aligned}
$$

In general, the zero-phase responses of the analysis filters can be expressed as follows:

$$
\begin{aligned}
& \widetilde{R}_{0}(\widetilde{x})=C_{0}\left[1+\sum_{n=1}^{L-1} \alpha_{0}(n) \widetilde{x}^{n}\right] \\
& \widetilde{R}_{1}(\widetilde{x})=C_{1}\left[1+\sum_{n=1}^{L} \alpha_{1}(n) \widetilde{x}^{n}\right] .
\end{aligned}
$$

For the sake of presentation, the causal counterparts of $\widetilde{R}_{0}(\widetilde{x})$ and $\widetilde{R}_{1}(\widetilde{x})$ are denoted respectively by $\widetilde{H}_{0}(\widetilde{z})$ and $\widetilde{H}_{1}(\widetilde{z})$, and are referred to as the prototype FB. With the following substitution of variable:

$$
\widetilde{x}=R_{Q}(x)=z^{G} Q\left(z^{2}\right)
$$




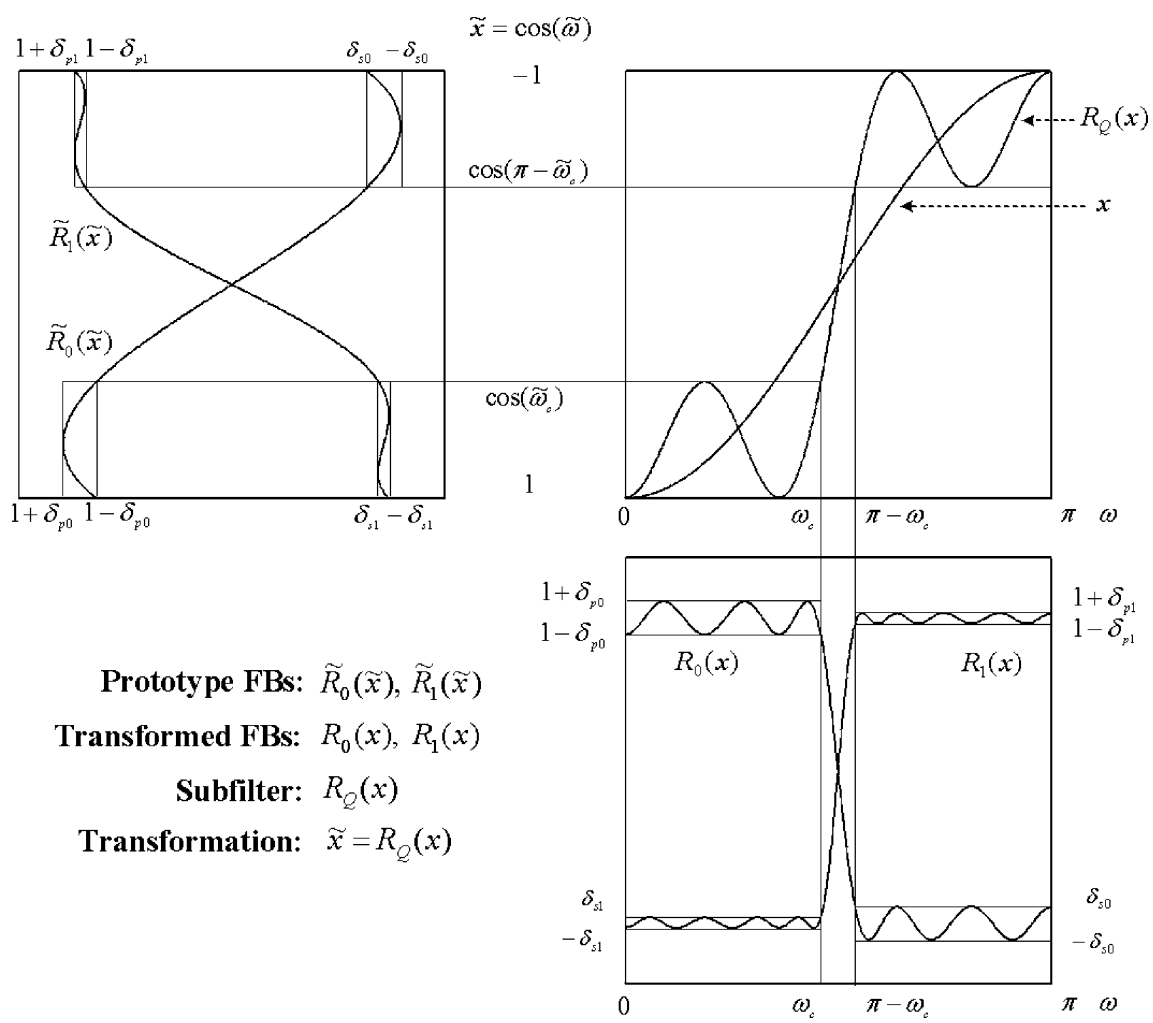

Fig. 3. General relations and specifications of the prototype FB, the subfilter and the multi-plet transformed FB.

where $R_{Q}(x)$ is the zero-phase response of the subfilter $Q\left(z^{2}\right)$ for some positive integer $G$, we obtain from (2-7) the zero-phase response of a new analysis filter pair as follows:

$$
\begin{aligned}
& R_{0}(x)=C_{0}\left[1+\sum_{n=1}^{L-1} \alpha_{0}(n)\left[R_{Q}(x)\right]^{n}\right] \text { and } \\
& R_{1}(x)=C_{1}\left[1+\sum_{n=1}^{L} \alpha_{1}(n)\left[R_{Q}(x)\right]^{n}\right] .
\end{aligned}
$$

We call the corresponding causal FB the transformed FB of the prototype. Since the transformed FB is obtained by using the substitution in (2-8), it can also be implemented by the same number of lifting steps as the prototype.

To analyze the effect of the transformation, let us express the $z$-transform variable of the prototype (transformed) FB as $\widetilde{z}=e^{j \tilde{\omega}}\left(z=e^{j \omega}\right)$, where $\widetilde{\omega}(\omega)$ is the digital radian frequency of the prototype (transformed) FB. From (2-8), the digital frequencies before and after transformation are related by $\widetilde{x}=\cos (\widetilde{\omega})=R_{Q}(x)=R_{Q}(\cos (\omega))$. Fig. 3 shows graphically the relationship of the various quantities in the transformation. It can be seen that $x$ varies monotonically from 1 to -1 as $\widetilde{\omega}$ varies from 0 to $\pi$, and the transition band of the prototype FB lies in the interval around $\widetilde{\omega}=\pi / 2$ or $\widetilde{x}=0$. Therefore, if $R_{Q}(x)$ is appropriately designed to have a sharper characteristics than $x=\cos (\omega)$ around $\omega=\pi / 2$, then the transformed FB will have much narrower transition band. Furthermore, if we want to preserve the passband and stopband ripples of the prototype FB in the transformed $\mathrm{FB}, R_{Q}(x)$ should map respectively the values of $x=\cos (\omega)$ in the passband and stopband of the prototype FB to the new passband and stopband of the transformed FB. It should be noted that the characteristics of $R_{Q}(x)$ need not be monotonic in these regions, while it is better to maintain a monotonic characteristic in the transition band of the final FB. Here, we shall employ an equiripple characteristic for $R_{Q}(x)$ in the passband/stopband of the final FB. This is similar to frequency transformation approach of designing odd-length two-channel linear-phase FIR FBs [6], [20], [27], except that we are now operating directly on the lifting structure of the prototype FB. It should be noted that $\widetilde{x}$ and $R_{Q}(x)$ can lie outside the interval $[-1,1]$ without affecting the PR condition of the transformed FB. However, one should check the magnitude response of the prototype FB outside the range $[-1,1]$ to ensure that significant degradation of the frequency characteristic of the transformed FB will not be introduced. For simplicity, we shall assume that $x$ lies inside the interval $[-1,1]$ in the rest of the paper.

Using these results, the design of a two-channel PR FB can be split into two much simpler sub-problems, namely the design of the prototype PR FB and the subfilter $R_{Q}(x)$. The advantage of this approach is that the length of the prototype PR FB, which is targeted to have rather wide transition bandwidth, are relatively short. Therefore, the number of variables, and hence the design complexity of the prototype FB can be greatly reduced, as compared to the direct design of the target FB using nonlinear constrained optimization with large number of variables in order to satisfy the tight specifications. On the other hand, the transformed FB can achieve a much narrower and prescribed transition bandwidth by properly designing the subfilter using conventional filter design technique as we shall see later in Sections IV and V, while preserving the passband/stopband ripples of the prototype FB. Next, we shall determine the specifications of the prototype FB and the subfilters. 


\section{Design Specifications and Methodology}

Obviously, the target specifications are identical to those of the transformed FB. Suppose that the specifications of the latter are as follows:

Low-Pass $: \begin{cases}1-\delta_{p 0} \leq R_{0}(x) \leq 1+\delta_{p 0}, & 0 \leq \omega \leq \omega_{c} \\ \delta_{s 0} \leq R_{0}(x) \leq \delta_{s 0}, & \pi-\omega_{c} \leq \omega \leq \pi\end{cases}$
High-Pass $: \begin{cases}1-\delta_{p 1} \leq R_{1}(x) \leq 1+\delta_{p 1}, & \pi-\omega_{c} \leq \omega \leq \pi \\ \delta_{s 1} \leq R_{1}(x) \leq \delta_{s 1}, & 0 \leq \omega \leq \omega_{c}\end{cases}$

where $\omega_{c}$ specifies the cutoff frequency; $\delta_{p i}$ and $\delta_{s i}$ are respectively the passband and stopband ripples of the analysis filters $H_{i}(z)$ and hence $R_{i}(x)$, for $i=0,1$. As mentioned earlier, the given specification can be met by first designing a prototype FB with the given passband and stopband ripples but with a wider transition band. After appropriate frequency transformation in (2-8), the given transition bandwidth can be achieved, while preserving the lifting structure, PR property, and passband/stopband ripples. Therefore, the specifications of the prototype FB can be expressed as

Low-Pass : $\begin{cases}1-\delta_{p 0} \leq \widetilde{R}_{0}(\widetilde{x}) \leq 1+\delta_{p 0}, & 0 \leq \widetilde{\omega} \leq \widetilde{\omega}_{c} \\ \delta_{s 0} \leq \widetilde{R}_{0}(\widetilde{x}) \leq \delta_{s 0}, & \pi-\widetilde{\omega}_{c} \leq \widetilde{\omega} \leq \pi\end{cases}$
High-Pass : $\begin{cases}1-\delta_{p 1} \leq \widetilde{R}_{1}(\widetilde{x}) \leq 1+\delta_{p 1}, & \pi-\widetilde{\omega}_{c} \leq \widetilde{\omega} \leq \pi \\ \delta_{s 1} \leq \widetilde{R}_{1}(\widetilde{x}) \leq \delta_{s 1}, & 0 \leq \widetilde{\omega} \leq \widetilde{\omega}_{c}\end{cases}$

where $\widetilde{\omega}_{c}$ is the cutoff frequency of the prototype FB. On the other hand, it can be seen from Fig. 3 that to transform the ripples of the prototype FB at the $\widetilde{\omega}$-domain to the frequency of interest at the $\omega$-domain, the subfilter should satisfy the following specifications:

$$
\begin{cases}\cos \left(\widetilde{\omega}_{c}\right) \leq R_{Q}(x) \leq 1, & 0 \leq \omega \leq \omega_{c} \\ -1 \leq R_{Q}(x) \leq-\cos \left(\widetilde{\omega}_{c}\right), & \pi-\omega_{c} \leq \omega \leq \pi\end{cases}
$$

If the prototype FB is monotonic decreasing at the stopband, say a maximally-flat function in [27], then an arbitrary small stopband attenuation can be achieved by choosing an appropriate subfilter that maps the value of the prototype FB near $\widetilde{x}= \pm 1$ to the stopband. The basic idea is similar to the design procedures of the structural PR FB in [1]-[4] and the triplet FB in [7]-[11], except that the prototype FBs in those FBs are fixed and have simple coefficients. In addition, since the design of those FBs is not viewed as a transformation, each subfilter is usually designed independently in order to obtain better performance. Alternatively, a set of prototype PR FBs with different passband/ stopband ripples can be designed offline. The subfilter can then be designed so that the transformed FB will be able to achieve a narrower transition bandwidth, while preserving the ripples of the prototype FB. Optimized prototype FBs usually lead to a better performance. It should be noted that the cutoff frequency of the prototype $\mathrm{FB}, \widetilde{\omega}_{c}$, in (2-11) and (2-12) can be freely chosen as long as it is larger than the desired cutoff frequency. Therefore, numerous combinations of the prototype FBs and the subfilters can be employed so as to satisfy the specifications in (2-10). The optimal combination can be determined by designing prototype PR FBs with different orders and select the one that yields the lowest complexity.

\section{FACTORIZATION OF PROTOTYPE FBS}

\section{A. Design of PR Prototype FBS}

As discussed in Section II, we can start with the design of a two-channel odd-length linear-phase $\operatorname{PR~FB~}\left(\widetilde{H}_{0}(\widetilde{z})\right.$ and $\left.\widetilde{H}_{1}(\widetilde{z})\right)$ which has a sufficiently high stopband attenuation but rather wide transition bandwidth. Therefore, the order of the analysis filters and the number of design variables required will be relatively small, making the nonlinear optimization much less complicated. With the choice of synthesis filters in $(2-2 b)$, the two-channel FB is PR if

$$
\widetilde{H}_{0}(-\widetilde{z}) \widetilde{H}_{1}(\widetilde{z})-\widetilde{H}_{0}(\widetilde{z}) \widetilde{H}_{1}(-\widetilde{z})=\widetilde{z}^{-n_{d}}
$$

where $n_{d}=\left(\widetilde{L}_{0}+\widetilde{L}_{1}\right) / 2-1$ for linear-phase FBs; $\widetilde{L}_{0}$ and $\widetilde{L}_{1}$ are respectively the lengths of $\widetilde{H}_{0}(z)$ and $\widetilde{H}_{1}(z)$. From the discussion in Section II-B, we shall consider $\widetilde{L}_{1}=\widetilde{L}_{0}+2$. In order to obtain a prototype FB with symmetric frequency responses, we consider the following objective function $\Phi$ :

$\min _{\boldsymbol{a}} \Phi=\sigma \delta_{0}+(1-\sigma) s$ subject to PR condition in (3-1),

where $\delta_{0}=\int_{0}^{\widetilde{\Omega}_{c}}|1-| \widetilde{H}_{0}\left(e^{j \tilde{\omega}}\right)||^{2} d \omega+\int_{\pi-\widetilde{\Omega}_{c}}^{\pi}\left|\widetilde{H}_{0}\left(e^{j \widetilde{\omega}}\right)\right|^{2} d \omega ;$ $\widetilde{\Omega}_{c}$ is the cutoff frequency of the prototype FB; $s=$ $\int_{0}^{\pi}|| \widetilde{H}_{0}\left(e^{j \widetilde{\omega}}\right)|-| \widetilde{H}_{1}\left(e^{j(\pi-\widetilde{\omega})}\right)||^{2} d \omega ; 0 \leq \sigma \leq 1$ controls the degree of similarity between $\widetilde{H}_{0}(\widetilde{z})$ and $\widetilde{H}_{1}(\widetilde{z})$; and $\boldsymbol{a}$ is the vector containing the filter coefficients of $\widetilde{H}_{0}(\widetilde{z})$ and $\widetilde{H}_{1}(\widetilde{z})$ (i.e., their impulse response coefficients). Here, $\widetilde{\Omega}_{c}$ is different from $\widetilde{\omega}_{c}$ defined in (2-10) since larger sidelobes usually occur near the band edges. Moreover, to design wavelet FBs, appropriate factors can be incorporated into $\mathscr{H}_{0}(\widetilde{z})$ and $\widetilde{H}_{1}(\widetilde{z})$ as shown in the following form:

$$
\begin{aligned}
\widetilde{H}_{0}(\widetilde{z}) & =\left(1+\widetilde{z}^{-1}\right)^{\widetilde{K}_{0}} P_{0}(\widetilde{z}) \text { and } \\
\widetilde{H}_{1}(\widetilde{z}) & =\left(1-\widetilde{z}^{-1}\right)^{\widetilde{K}_{1}} P_{1}(\widetilde{z})
\end{aligned}
$$

where $\widetilde{K}_{0}$ and $\widetilde{K}_{1}$ are the number of zeros to be imposed respectively at $\widetilde{\omega}=\pi$ and $\widetilde{\omega}=0$ for $\widetilde{H}_{0}(\widetilde{z})$ and $\widetilde{H}_{1}(\widetilde{z})$. The above design problem is a constrained nonlinear optimization problem, which can be solved by the NCONF/DCONF subroutine in the IMSL library. Since $\widetilde{H}_{0}(\widetilde{z})$ and $\widetilde{H}_{1}(\widetilde{z})$ are linear-phase FIR filters, the number of variables can be reduced approximately by a factor of two. In addition, because of the relaxed specifications (i.e., wide transition bandwidth), the performance of the prototype FBs can easily be controlled although the design problem still requires nonlinear optimization. It should be noted that a set of prototype FBs can be designed offline so that they can be transformed to meet different specifications.

\section{B. Factorization Using Euclidean Algorithm}

For completeness, we shall briefly summarize the factorization of the linear-phase prototype PR FB using the Euclidean algorithm [16], [17]. The factorization of general two-channel 
PR FB can be found in [12], [13]. Suppose that the $z$-transform of $\widetilde{H}_{i}(\widetilde{z}), i=0,1$, is given by

$$
\widetilde{H}_{i}(\widetilde{z})=\sum_{n=0}^{\widetilde{L}_{i}-1} \widetilde{h}_{i}(n) \widetilde{z}^{-n}
$$

then its zero-phase response can be expressed as follows:

$$
\begin{aligned}
\widetilde{R}_{i}(\widetilde{z}) & =\widetilde{h}_{i}\left(M_{i}\right)+\sum_{n=1}^{M_{i}} \widetilde{h}_{i}\left(M_{i}-n\right)\left[\widetilde{z}^{n}+\widetilde{z}^{-n}\right] \\
& =\widetilde{h}_{i}\left(M_{i}\right)+\sum_{n=1}^{M_{i}} 2 \widetilde{h}_{i}\left(M_{i}-n\right) T_{n}[\cos (\widetilde{\omega})],
\end{aligned}
$$

where $M_{i}=\left(\widetilde{L}_{i}-1\right) / 2, \widetilde{z}^{n}+\widetilde{z}^{-n}=2 \cos (n \widetilde{\omega})=2 T_{n}[\cos (\widetilde{\omega})]$ and $T_{n}[\bullet]$ is the $n$ th-order Chebyshev polynomial. Substituting $\widetilde{x}=\cos (\widetilde{\omega})$ into $(3-5)$, one gets

$$
\widetilde{R}_{i}(\widetilde{x})=\sum_{n=0}^{M_{i}} b_{i}(n) \widetilde{x}^{n}
$$

where $b_{i}(n)$ is related to $\widetilde{h}_{i}(n)$ and the coefficients of the Chebyshev polynomials. Comparing (3-6) with (2-7), we obtain $C_{i} \alpha_{i}(n)=b_{i}(n)$ for $n=1,2, \ldots, M_{i}$, and the scaling constants are given by

$$
C_{i}=b_{i}(0)
$$

To simplify notation, we normalize $\widetilde{R}_{0}(\widetilde{x})$ and $\widetilde{R}_{1}(\widetilde{x})$ by $C_{0}$ and $C_{1}$, respectively, and let

$$
A_{0}(\widetilde{x})=\frac{\widetilde{R}_{1}(\widetilde{x})}{C_{1}} \text { and } A_{1}(\widetilde{x})=\frac{\widetilde{R}_{0}(\widetilde{x})}{C_{0}} .
$$

From the PR condition in (3-1), $\widetilde{H}_{0}(-\widetilde{z}) \widetilde{H}_{1}(\widetilde{z})$ (or $\left.\widetilde{H}_{0}(\widetilde{z}) \widetilde{H}_{1}(-\widetilde{z})\right)$ has to be a halfband filter and hence $A_{0}(\widetilde{x}) A_{1}(-\widetilde{x})$ (or $A_{0}(-\widetilde{x}) A_{1}(\widetilde{x})$ ) is also a zero-phase halfband filter. In other words, all even-indexed coefficients of $A_{0}(\widetilde{x}) A_{1}(-\widetilde{x})$ (or $A_{0}(-\widetilde{x}) A_{1}(\widetilde{x})$ ) are zero except for its constant term. To illustrate the idea of the Euclidean Algorithm, consider the zero-phase halfband filter $A_{0}(\widetilde{x}) A_{1}(-\widetilde{x})$, where $\operatorname{deg}\left[\widetilde{R}_{1}(\widetilde{x})\right]-\operatorname{deg}\left[\widetilde{R}_{0}(\widetilde{x})\right]=1$ (i.e., $M_{1}-M_{0}=1$ and $\left.\widetilde{L}_{1}-\widetilde{L}_{0}=2\right)$. According to (3-8), suppose that $A_{i}(\widetilde{x})$ is given by $A_{i}(\widetilde{x})=1+\sum_{n=1}^{M_{1-i}} a_{i}(n) \widetilde{x}^{n}$, for $i=0,1$, then one obtains

$$
a_{0}\left(M_{0}+1\right) a_{1}\left(M_{0}-1\right)-a_{0}\left(M_{0}\right) a_{1}\left(M_{0}\right)=0
$$

because the coefficient of $\widetilde{x}^{2 M_{0}}$ (the second largest power of $\widetilde{x}$ ) of $A_{0}(\widetilde{x}) A_{1}(-\widetilde{x})$ must be zero. Now consider another polynomial $A_{2}(\widetilde{x})=A_{0}(\widetilde{x})-\beta \widetilde{x} A_{1}(\widetilde{x})$ with a constant $\beta$ such that

$$
a_{0}\left(M_{0}+1\right)-\beta a_{1}\left(M_{0}\right)=0
$$

(i.e., the term $\widetilde{x}^{M_{0}+1}$ is removed), then, using (3-9) and (3-10), the coefficient of $\widetilde{x}^{M_{0}}$ is equal to

$$
a_{0}\left(M_{0}\right)-\beta a_{1}\left(M_{0}-1\right)=0
$$

and hence $\operatorname{deg}\left[A_{2}(\widetilde{x})\right]=\operatorname{deg}\left[A_{1}(\widetilde{x})\right]-1=\operatorname{deg}\left[A_{0}(\widetilde{x})\right]-2$. Also, it can be seen that $A_{2}(\widetilde{x}) A_{1}(-\widetilde{x})=\left[A_{0}(\widetilde{x})-\right.$ $\left.\beta x A_{1}(\widetilde{x})\right] A_{1}(-\widetilde{x})$ (or $A_{2}(-\widetilde{x}) A_{1}(\widetilde{x})$ ) form another zero-phase halfband filter because of the fact that all the even-indexed coefficients of $\widetilde{x} A_{1}(\widetilde{x}) A_{1}(-\widetilde{x})$ are zero. Consequently, similar results can also be applied to the division of $A_{1}(\widetilde{x})$ by $A_{2}(\widetilde{x})$. Repeating this procedure gives rise to the Euclidean algorithm. More precisely, the basic idea of the algorithm is summarized as follows:

$A_{m}(\widetilde{x})=A_{m+1}(\widetilde{x}) V_{m}(\widetilde{x})+A_{m+2}(\widetilde{x}), \quad m=0,1, \ldots, L-1$

where $V_{m}(\widetilde{x})$ and $A_{m+2}(\widetilde{x})$ are respectively the quotient and remainder; and $L$ is the number of lifting steps

$$
L=M_{0}+1=\operatorname{deg}\left[\widetilde{R}_{0}(\widetilde{x})\right]+1 .
$$

Note that in the last step $(m=L-1)$, the division in (3-12) should $\operatorname{read} A_{L-1}(\widetilde{x})=V_{L-1}(\widetilde{x})+1$ (i.e., $\left.A_{L}(\widetilde{x})=A_{L+1}(\widetilde{x})=1\right)$. The lifting coefficients $p_{m}$ and the quotients $V_{m}(\widetilde{x})$ are related by

$$
V_{L-1-m}(\widetilde{x})=p_{m} \cdot \widetilde{x}, \quad \text { for } m=0,1, \ldots, L-1 \text {. }
$$

Once the constant parameters $C_{0}, C_{1}$ and $p_{m}$ for $m=$ $0,1, \ldots, L-1$ are obtained, the prototype FB can be implemented as the lifting structure shown in Fig. 2. As an example, consider the structure of the PR FB used in [1]. With identical subfilters of the form $\left(1+\widetilde{z}^{-1}\right) / 2$, the $z$-transforms of the analysis filter pair are given by $\widetilde{H}_{0}(\widetilde{z})=(1 / 4)\left(1+2 \widetilde{z}^{-1}+\widetilde{z}^{-2}\right)$ and $H_{1}(\widetilde{z})=(1 / 8)\left(-1-2 \widetilde{z}^{-1}+6 \widetilde{z}^{-2}-2 \widetilde{z}^{-3}-\widetilde{z}^{-4}\right)$. From these, we obtain $A_{0}(\widetilde{x})=1+0.5 \widetilde{x}+0.5 \widetilde{x}^{2}, A_{1}(\widetilde{x})=1+\widetilde{x}$, $C_{0}=0.5$ and $C_{1}=1$. Using the abovementioned Euclidean algorithm, two division steps can be performed as follows: $A_{0}(\widetilde{x})=-0.5 \widetilde{x} A_{1}(\widetilde{x})+1=p_{1} \widetilde{x} A_{1}(\widetilde{x})+A_{2}(\widetilde{x})$ and $A_{1}(\widetilde{x})=\widetilde{x} A_{2}(\widetilde{x})+1=p_{0} \widetilde{x} A_{2}(\widetilde{x})+A_{3}(\widetilde{x})$, where $A_{2}(\widetilde{x})=A_{3}(\widetilde{x})=1, p_{0}=1$ and $p_{1}=-0.5$.

In general, if $\operatorname{deg}\left[\widetilde{R}_{1}(\widetilde{x})\right]-\operatorname{deg}\left[\widetilde{R}_{0}(\widetilde{x})\right]=2 d-1$ (or $\widetilde{L}_{1}=$ $\widetilde{L}_{0}+4 d-2$ ), where $d$ is a positive integer, then the quotient $V_{m}(\widetilde{x})$ becomes

$$
V_{0}(\widetilde{x})=\sum_{n=0}^{d-1} v(n) \widetilde{x}^{2 n+1} \text { and } V_{m}(\widetilde{x})=p_{L-1-m} \cdot \widetilde{x}
$$

for $m=1,2, \ldots, L-1$. An example for $d=3$ can be found in [16]. Since $V_{0}(\widetilde{x})$ is also a function of $\widetilde{x}$, extension of our approach to this case is straightforward. For simplicity, only the case in (3-14) (i.e., $d=1$ ) is considered in this paper. Next, we shall consider the design of the subfilters using SOCP.

\section{Design OF FIR Subfilters Using SOCP}

\section{A. SOCP Formulation}

Although our discussion so far regarding the subfilter is focused on linear-phase FIR filters, we found that the proposed transformation method works well for approximately linear-phase subfilters. More precisely, $\widetilde{x}$ in (2-8) is now transformed to $R_{Q}(x)$, which is a complex quantity. However, it has a higher order than a linear-phase subfilter with the same passband delay in order to give a better approximation to the 
transformation characteristics. This is similar to the low-delay structural PR FBs in [2] and [4], and the triplet FB in [10] and [11], where the subfilters are chosen as low-delay, instead of linear-phase, FIR filters. The advantage over their linear-phase counterparts is that the overall system delay of the transformed FBs can be reduced.

To begin with, denote the transfer function of the FIR subfilter to be designed by

$$
Q(z)=\sum_{n=0}^{L_{Q}-1} q(n) z^{-n}
$$

where $L_{Q}$ and $q(n)$ are respectively the length and the impulse response of the subfilter. By introducing a delay term $e^{-j \omega G}$ into (2-12), the desired response of the subfilter can be rewritten as

$$
Q_{d}(\omega)=m \cdot e^{-j \omega G / 2}, \quad 0 \leq \omega \leq 2 \omega_{c}
$$

where $m=\left[1+\cos \left(\widetilde{\omega}_{c}\right)\right] / 2$ with the maximum allowable ripple $\delta_{Q}=\left[1-\cos \left(\widetilde{\omega}_{c}\right)\right] / 2$. From (4-1) and (4-2), we can see that $G$ is given by

$$
G=L_{Q}-1-D
$$

where $D$ is a delay reduction parameter. When $D=0$, the subfilter is an even-length linear-phase FIR filter with symmetric impulse response because of the specification in (2-12). Consequently, $G$ has to be an odd positive integer. Moreover, it is observed that $Q(z)$ is related to a linear-phase halfband filter $A(z)$ of the form $A(z)=0.5\left[m^{-1} Q\left(z^{2}\right)+z^{-G}\right]$ with identical passband and stopband ripples. Since $\delta_{Q}$ is twice of the ripple error of $A(z)$, the Kaiser's formula [30] can therefore be used to estimate the length of $A(z)$ (denoted by $L_{A}$ ) and $L_{Q}$

$$
L_{A} \approx \frac{-20 \log _{10}\left(\frac{\delta_{Q}}{2}\right)-13}{2.324 \cdot\left(\pi-2 \omega_{c}\right)}+1
$$

and hence we have $L_{Q} \approx\left(L_{A}+3\right) / 2$. This provides a good starting point to determine $L_{Q}$ to meet a given specification as shown in (2-12). For the low-delay case, $L_{Q}$ can be chosen as either odd or even integer, provided that $G$ is an odd integer, and $D$ is a positive integer corresponding to the amount of delay reduction. From (4-4), the estimated value of $L_{Q}$ is increased until the required specification is met, since slightly longer subfilter length is usually required as compared to its linear-phase counterparts with the same specification.

For a given subfilter length, the design problem is to approximate the desired response $Q_{d}(\omega)$ by $Q\left(e^{j \omega}\right)$ in the minimax sense by minimizing

$$
E_{\infty}=\max _{\omega}\left|Q\left(e^{j \omega}\right)-Q_{d}(\omega)\right|, \omega \in\left[0,2 \omega_{c}\right]
$$

We shall formulate this problem as a convex programming problem and solve it using SOCP [22]-[25]. The advantage of formulating the objective function as a convex problem such as SOCP is that the resulting problem is convex and the optimal solution, if it exists, can be found. In addition, other linear equalities can easily be incorporated to obtain wavelet bases, as we shall illustrate later in this Section. Alternatively, (4-5) can be formulated as a semi-definite programming (SDP) problem [4], [10], [11], [26], which may provide more flexibility but requires a longer design time. For simplicity, only the SOCP formulation is considered below. To formulate the minimization problem of $E_{\infty}$ as a SOCP problem, $Q\left(e^{j \omega}\right)$ is first expressed in terms of the design variables $\boldsymbol{q}=\left[q(0), \ldots, q\left(L_{Q}-1\right)\right]^{T}$ as

$$
Q\left(e^{j \omega}\right)=\sum_{n=0}^{L_{Q}-1} q(n) e^{-j \omega n}=\boldsymbol{q}^{T}\{\boldsymbol{c}(\omega)+j \boldsymbol{s}(\omega)\}
$$

where $\boldsymbol{c}(\omega)=\operatorname{Re}[\boldsymbol{e}(\omega)], \boldsymbol{s}(\omega)=\operatorname{Im}[\boldsymbol{e}(\omega)] ; \operatorname{Re}[\bullet]$ and $\operatorname{Im}[\bullet]$ denote respectively the real and imaginary parts of the elements inside the square bracket; and $\boldsymbol{e}(\omega)=\left[1, e^{-j \omega} \ldots, e^{-j\left(L_{Q}-1\right) \omega}\right]^{T}$. Hence, (4-5) can be rewritten as follows:

$$
\min _{\boldsymbol{q}} \delta \quad \text { subject to } \delta-\left[\alpha_{R}^{2}(\omega)+\alpha_{I}^{2}(\omega)\right]^{1 / 2} \geq 0
$$

for $\omega \in\left[0,2 \omega_{c}\right]$, where $\alpha_{R}(\omega)=\boldsymbol{q}^{T} \boldsymbol{c}(\omega)-\operatorname{Re}\left[Q_{d}(\omega)\right]$ and $\alpha_{I}(\omega)=\boldsymbol{q}^{T} \boldsymbol{s}(\omega)-\operatorname{Im}\left[Q_{d}(\omega)\right]$. Discretizing the frequency variable $\omega$ over a dense set of frequencies $\left\{\omega_{i}, 1 \leq i \leq K_{\omega}\right\}$ in the range of interest, the constraints in (4-7) becomes $\bar{\delta}-\left[\alpha_{R}^{2}\left(\omega_{i}\right)+\right.$ $\left.\alpha_{I}^{2}\left(\omega_{i}\right)\right]^{1 / 2} \geq 0$. Moreover, by defining the augmented variable $\boldsymbol{y}=\left[\begin{array}{ll}\delta & \boldsymbol{q}^{T}\end{array}\right]^{T}$,(4-7) can be cast to the following standard SOCP problem:

$$
\min _{\boldsymbol{y}} \boldsymbol{d}^{T} \boldsymbol{y} \quad \text { subject to } \boldsymbol{d}^{T} \boldsymbol{y} \geq\left\|\boldsymbol{U}_{i} \boldsymbol{y}-\boldsymbol{v}_{i}\right\|_{2}
$$

where $\boldsymbol{d}=\left[\begin{array}{ll}1 & O_{L_{Q}}^{T}\end{array}\right]^{T}, \boldsymbol{U}_{i}=\left[\begin{array}{ll}0 & \boldsymbol{c}\left(\omega_{i}\right)^{T} \\ 0 & \boldsymbol{s}\left(\omega_{i}\right)^{T}\end{array}\right], \boldsymbol{v}_{i}=$ $\left[\begin{array}{l}\operatorname{Re}\left[Q_{d}\left(\omega_{i}\right)\right] \\ \operatorname{Im}\left[Q_{d}\left(\omega_{i}\right)\right]\end{array}\right] ;\|\cdot\|_{2}$ denotes the Euclidean norm; and $O_{N}$ is a $N \times 1$ zero vector. A similar formulation for the least squares (LS) criterion can also be derived.

\section{B. Imposing the K-Regularity Condition}

To construct a wavelet FB, the analysis filter pair $H_{0}(z)$ and $H_{1}(z)$ should possess at least one zero at $\omega=\pi$ and $\omega=0$, respectively. Let $K_{0}$ and $K_{1}$ (called the $K$-regularity) be the number of zeros to be imposed respectively at $\omega=\pi$ and $\omega=0$ for $H_{0}(z)$ and $H_{1}(z)$ with $K_{0} \geq K_{1} \geq 1$. Hence, the $K$-regularity condition can be written as follows:

$$
\left[\frac{d^{k_{0}}}{d \omega^{k_{0}}} H_{0}\left(e^{j \omega}\right)\right]_{\omega=\pi}=\left[\frac{d^{k_{1}}}{d \omega^{k_{1}}} H_{1}\left(e^{j \omega}\right)\right]_{\omega=0}=0
$$

for $k_{0}=0, \ldots, K_{0}-1$ and $k_{1}=0, \ldots, K_{1}-1$. Furthermore, let $r_{0}$ and $r_{1}$ be the roots of $\widetilde{R}_{0}(\widetilde{x})=0$ and $\widetilde{R}_{1}(\widetilde{x})=0$, respectively. In general, more than one such solution may exist. We are only interested in those roots which lie inside the stopband region of the prototype FB. More precisely, we have

$$
-1 \leq r_{0} \leq-\cos \left(\widetilde{\omega}_{c}\right), \text { and } \cos \left(\widetilde{\omega}_{c}\right) \leq r_{1} \leq 1
$$


After the transformation of variable, the number of zeros imposed for the analysis filters is closely related to the filters with the following form:

$$
H_{Q 0}(z)=Q\left(z^{2}\right)-r_{0} z^{-G} \text { and } H_{Q 1}(z)=Q\left(z^{2}\right)-r_{1} z^{-G} \text {. }
$$

Therefore, the $K$-regularity condition in (4-9) is equivalent to

$$
\left[\frac{d^{k_{0}}}{d \omega^{k_{0}}} H_{Q 0}\left(e^{j \omega}\right)\right]_{\omega=\pi}=\left[\frac{d^{k_{1}}}{d \omega^{k_{1}}} H_{Q 1}\left(e^{j \omega}\right)\right]_{\omega=0}=0
$$

for $k_{0}=0, \ldots, K_{0}-1$ and $k_{1}=0, \ldots, K_{1}-1$. However, it is noticed that the constraints in (4-12) are contradictory to each other due to the upsampling operation and odd positive integer $G$. For example, imposing one zero on $H_{Q 0}(z)$ at $\omega=\pi$ implies that $Q\left(z^{2}\right)+r_{0} z^{-G}$ also have one zero at $\omega=0$. Since $r_{0}$ and $r_{1}$ are of different signs according to (4-10), the constraints in (4-12) cannot be satisfied simultaneously. As a result, we further require $r_{0}$ and $r_{1}$ to satisfy the following condition:

$$
r_{0}=-r_{1}=r
$$

Equations (4-10) and (4-13) give us the general conditions for constructing a wavelet $\mathrm{FB}$, even if the prototype $\mathrm{FB}$ is not a wavelet FB. It should be noted that the prototype FBs considered in [1]-[10] are indeed wavelet FBs with $K$-regularity of certain orders. In this case, we have $r=-1$, and are able to construct a wavelet FB by imposing a flatness of prescribed order to each subfilter (since the subfilter at each lifting step may be different) at $\omega=0$. With (4-10) and (4-13), the $K$-regularity condition can be simplified to

$$
\left[\frac{d^{k}}{d \omega^{k}} H_{Q}\left(e^{j \omega}\right)\right]_{\omega=\pi}=0, \quad \text { for } k=0, \ldots, K_{Q}-1
$$

where $H_{Q}(z)=Q\left(z^{2}\right)-r z^{-G}$. It can be seen that if $H_{Q}\left(e^{j \omega}\right)$ has $K_{Q}$ zeros at $\omega=\pi$, then $H_{0}(z)$ and $H_{1}(z)$ also have $K_{0}=$ $K_{Q}$ and $K_{1}=K_{Q}$ zeros at $\omega=\pi$ and $\omega=0$, respectively. Expanding (4-14) and after slight manipulation, one gets a set of linear equality constraints as follows:

$$
\sum_{n=0}^{L_{Q}-1}(2 n)^{k} q(n)=-r G^{k}, \quad k=0, \ldots, K_{Q}-1
$$

and its matrix form is given by

$$
\boldsymbol{A} \cdot \boldsymbol{q}=\boldsymbol{b}
$$

where $[\boldsymbol{A}]_{k, n}=(2 n)^{k}$ and $[\boldsymbol{b}]_{k}=-r G^{k}$ for $k=0, \ldots, K_{Q}-1$ and $n=0, \ldots, L_{Q}-1$. Here, $[\boldsymbol{A}]_{k, n}$ denotes the $(k, n)$ th entry of matrix $\boldsymbol{A}$. Under the SOCP framework, these additional linear equality constraints can be easily incorporated.

\section{DESIGN OF FIR SUBFILTERS USING SOCP}

In this section, the subfilter is assumed to be the following IIR function:

$$
R_{m}(z)=\frac{G_{m}(z)}{D(z)}=\frac{\sum_{n=0}^{L_{G, m}-1} g_{m}(n) z^{-n}}{\sum_{n=0}^{L_{D}-1} d(n) z^{-n}}, \quad d(0)=1
$$

where $L_{G, m}$ and $L_{D}$ are, respectively, the lengths of numerator and denominator of $R_{m}(z)$ with $L_{G, m} \geq L_{D}$. To satisfy the specification in (2-12), we shall employ the SOCP-based model reduction technique proposed in [18] and [19], which extends the method in [31], to convert the desired FIR subfilter $Q(z)$ to an IIR filter $R_{m}(z)$ in the form of (5-1). More precisely, the common denominator $D(z)$ is first determined using a simple iterative design procedure, given a target FIR function. Once the denominator is obtained, the numerator $G_{m}(z)$ is then designed in LS sense optimally using convex programming, say SOCP, where linear equalities or convex quadratic constraints can easily be imposed to further improve the frequency characteristic of the resulting IIR filter. Apart from its simple design procedure, the advantage of the model reduction approach in [18] and [19] is that the resulting IIR filter is guaranteed to be stable, and the frequency characteristics such as the magnitude and phase responses of the FIR prototype filter is well preserved. In order to approximate $Q(z)$ with small enough errors using the technique in [18] and [19], we found that the length of the denominator of $R_{m}(z)$ should satisfy the following condition:

$$
L_{D} \geq\lceil\operatorname{grd}(Q)\rceil+1
$$

where $\operatorname{grd}(y)$ is the passband group delay of the FIR function $y(z)$ and $\lceil w\rceil$ denotes the integer just larger than or equal to $w$. In other words, according to (4-3), we have

$$
L_{D} \geq \frac{(G+1)}{2}+1
$$

This tells us that the savings in number of multiplications and additions would be substantial if model reduction is applied to FIR functions with lower system delay. Therefore, it is preferable to use IIR filters when low system delay and low arithmetic complexity are of interest. In what follows, we shall mainly focus on the design of the numerator. Interested readers are referred to [18], [19] and [31] for the determination of $D(z)$.

After $D\left(e^{j \omega}\right)$ is designed, $R_{m}\left(e^{j \omega}\right)$ in (5-1) can be rewritten more compactly in matrix form of the design variables $\boldsymbol{g}_{m}=$ $\left[g_{m}(0), \ldots, g_{m}\left(L_{G, m}-1\right)\right]^{T}$ as follows:

$$
R_{m}\left(e^{j \omega}\right)=\boldsymbol{g}_{m}^{T}\left[\boldsymbol{c}_{m}(\omega)+j \boldsymbol{s}_{m}(\omega)\right]
$$

where $\boldsymbol{c}_{m}(\omega)=\operatorname{Re}\left[\boldsymbol{e}_{m}(\omega) / D\left(e^{j \omega}\right)\right], \boldsymbol{s}_{m}(\omega)=\operatorname{Im}\left[\boldsymbol{e}_{m}(\omega)\right.$ $\left./ D\left(e^{j \omega}\right)\right]$ and $e_{m}(\omega)=\left[1, e^{-j \omega} \ldots, e^{-j\left(L_{G, m}-1\right) \omega}\right]^{T}$. To approximate the response of $Q(z)$ by $G_{m}(z)$, given $D(z)$, in the LS sense, we have

$$
R_{m}\left(e^{j \omega}\right)=\boldsymbol{g}_{m}^{T}\left[\boldsymbol{c}_{m}(\omega)+j \boldsymbol{s}_{m}(\omega)\right]
$$

where $\boldsymbol{U}_{m}=\int_{-\pi}^{\pi}\left[\boldsymbol{c}_{m}(\omega) \boldsymbol{c}_{m}(\omega)^{T}+\boldsymbol{s}_{m}(\omega) \boldsymbol{s}_{m}(\omega)^{T}\right] d \omega, \boldsymbol{v}_{m}=$ $\int_{-\pi}^{\pi}\left\{\boldsymbol{c}_{m}(\omega) \operatorname{Re}\left[Q\left(e^{j \omega}\right)\right]+\boldsymbol{s}_{m}(\omega) \operatorname{Im}\left[Q\left(e^{j \omega}\right)\right]\right\} d \omega$, and $k_{m}=$ $\int_{-\pi}^{\pi}\left|Q\left(e^{j \omega}\right)\right|^{2} d \omega$. To formulate the minimization problem of $E_{L S}\left(g_{m}\right)$ as a SOCP problem, (5-5) is rewritten as follows:

$$
E_{L S}\left(\boldsymbol{g}_{m}\right)=\left\|\boldsymbol{U}_{m}^{1 / 2} \boldsymbol{g}_{m}-\boldsymbol{U}_{m}^{-1 / 2} \boldsymbol{v}_{m}\right\|_{2}^{2}-\left(\boldsymbol{v}_{m}^{T} \boldsymbol{U}_{m}^{-1} \boldsymbol{v}_{m}-k_{m}\right) \text {. }
$$


Moreover, by defining the augmented variable $\boldsymbol{x}_{m}=$ $\left[\begin{array}{ll}\delta_{m} & \boldsymbol{g}_{m}^{T}\end{array}\right]^{T}$, the LS design problem can be reformulated as the following SOCP:

$$
\min _{\boldsymbol{x}_{m}} \boldsymbol{h}_{m}^{T} \boldsymbol{x}_{m} \quad \text { subject to } \boldsymbol{h}_{m}^{T} \boldsymbol{x}_{m} \geq\left\|\overline{\boldsymbol{U}}_{m} \boldsymbol{x}_{m}-\overline{\boldsymbol{v}}_{m}\right\|_{2}
$$

where $\boldsymbol{h}_{m}=\left[\begin{array}{ll}1 & \boldsymbol{O}_{L_{G, m}}^{T}\end{array}\right]^{T}, \overline{\boldsymbol{U}}_{m}=\left[\begin{array}{ll}\boldsymbol{O}_{L_{G, m}} & \boldsymbol{U}_{m}^{1 / 2}\end{array}\right]$ and $\overline{\boldsymbol{v}}_{m}=\boldsymbol{U}_{m}^{-1 / 2} \boldsymbol{v}_{m}$.

As discussed in Section IV-B, it is possible to construct wavelet bases by imposing certain zeros at $\omega=\pi$ to the filter $H_{Q}(z)$ associated with the subfilter $Q(z)$. Replacing $Q(z)$ in (4-14) by $R_{m}(z)$, one gets the following condition for the transformed FB to satisfy the $K$-regularity condition:

$$
\left[\frac{d^{k}}{d \omega^{k}} H_{R_{m}}\left(e^{j \omega}\right)\right]_{\omega=\pi}=0
$$

for $k=0, \ldots, K_{R_{m}}-1$ and $m=0, \ldots, L-1$. It can be seen that if $H_{R_{m}}\left(e^{j \omega}\right)$ has $K_{R_{m}}$ zeros at $\omega=\pi$, then $H_{0}(z)$ and $H_{1}(z)$ also have $K_{0}=\min \left\{K_{R_{m}}, m=0,1, \ldots, L-2\right\}$ and $K_{1}=\min \left\{K_{R_{m}}, m=0,1, \ldots, L-1\right\}$ zeros at $\omega=\pi$ and $\omega=0$, respectively. To impose $K_{R_{m}}$ zeros on $H_{R_{m}}\left(e^{j \omega}\right)$ at $\omega=\pi$, the coefficients of $G_{m}(z)$ should satisfy the following linear equality constraints:

$$
\sum_{n=0}^{L_{G, m}-1}(2 n)^{k} g_{m}(n)=-r \sum_{n=0}^{L_{D}-1}(G+2 n)^{k} d(n)
$$

for $k=0, \ldots, K_{R_{m}}-1$ and $m=0, \ldots, L-1$. Using the SOCP framework, it is very effective and convenient to impose these additional linear constraints on the subfilters because $d(n)$ are known. To construct causal stable IIR multi-plet wavelet bases, identical subfilter satisfying (5-8) can be employed.

However, since the numerator is designed with LS design criterion after determining the denominator, larger ripple error is usually encountered near the band-edge of the model-reduced filter, which in turn degrades the stopband attenuation of the transformed FB. Therefore, to improve the frequency characteristic, additional constraints on the stopband ripples are imposed

$$
\left|H_{i}\left(e^{j \omega}\right)\right| \leq \varepsilon_{i}, \quad \text { for } \omega \text { in the stopband, } i=0,1
$$

where $\varepsilon_{i}$ is the prescribed peak stopband ripple to be imposed on the analysis filters $H_{i}(z)$. To simplify the design, the first $L-2$ subfilters (denoted by $R_{m}(z)=R(z)=G(z) / D(z)$, $m=0,1, \ldots, L-3)$ are obtained by model reducing its FIR counterpart using the model reduction technique in [17], while the numerators of the remaining two subfilters are separately designed to satisfy the constraints in (5-10). Replacing $Q_{m}(z)$ in (2-1) with $R_{m}(z)$ and substituting into (2-2), the peak stopband constraint in (5-10) can be rewritten as follows:

$$
\varepsilon_{i}-\left[\gamma_{R, i}^{2}(\omega)+\gamma_{I, i}^{2}(\omega)\right]^{1 / 2} \geq 0, \omega \text { in the stopband }
$$

where $\gamma_{R, i}(\omega)=\boldsymbol{g}_{L-2+i}^{T} \operatorname{Re}\left[\boldsymbol{y}_{i}(\omega)\right]+\operatorname{Re}\left[e^{-j 2 N_{L-2+i}}\right.$. $\left.H^{(L-4+i)}\left(e^{j \omega}\right)\right], \gamma_{I, i}(\omega)=\boldsymbol{g}_{L-2+i}^{T} \operatorname{Im}\left[\boldsymbol{y}_{i}(\omega)\right]+\operatorname{Im}\left[e^{-j 2 N_{L-2+i}}\right.$. $\left.H^{(L-4+i)}\left(e^{j \omega}\right)\right], \boldsymbol{y}_{i}(\omega)=e^{-j 2 \omega N_{L-2+i}} \cdot H^{(L-3+i)}\left(e^{j \omega}\right) \cdot$ $\boldsymbol{e}_{L-2+i}(2 \omega)$; and $\boldsymbol{e}_{L-2+i}(\omega)$ is defined in (5-4). Note, $H_{0}(z)$ and $H_{1}(z)$ are designed in turn, i.e., $i=0$ and then $i=1$ in
(5-11). Moreover, in the design problem for each value of $i$, $G_{m}(z), m=0,1, \ldots, L-3+i$, and hence $H^{(L-4+i)}\left(e^{j \omega}\right)$ and $H^{(L-3+i)}\left(e^{j \omega}\right)$ are known. Therefore, $(5-11)$ is a quadratic inequality constraint in $\boldsymbol{g}_{L-2+i}$ and can be rewritten as the following SOCP constraint:

$$
\varepsilon_{i} \geq\left\|\boldsymbol{R}_{i}(\omega) \boldsymbol{x}_{L-2+i}+\boldsymbol{f}_{i}(\omega)\right\|_{2}, \omega \text { in the stopband (5-12) }
$$

where $\boldsymbol{R}_{i}(\omega)=\left[\begin{array}{ll}0, & \operatorname{Re}\left[\boldsymbol{y}_{i}^{T}(\omega)\right] \\ 0, & \operatorname{Im}\left[\boldsymbol{y}_{i}^{T}(\omega)\right]\end{array}\right]$ and $\boldsymbol{f}_{i}(\omega)=$
$\left[\operatorname{Re}\left[e^{-j 2 N_{L-2+i}} \cdot H^{(L-4+i)}\left(e^{j \omega}\right)\right]\right.$ $\left[\begin{array}{l}\operatorname{Re}\left[e^{-j 2 N_{L-2+i}} \cdot H^{(L-4+i)}\left(e^{j \omega}\right)\right] \\ \operatorname{Im}\left[e^{-j 2 N_{L-2+i}} \cdot H^{(L-4+i)}\left(e^{j \omega}\right)\right]\end{array}\right]$. By discretizing

in the stopband region, these constraints on the peak ripples can be augmented to the existing SOCP constraints in (5-7) for determining $G_{L-2+i}(z)$. Note that it is also possible to obtain a wavelet base if $G(z), G_{L-2}(z)$ and $G_{L-1}(z)$ are designed to satisfy (5-9) simultaneously. A similar formulation for the minimax criterion can also be derived. We now present several design examples.

\section{DESIGN EXAMPLES}

In all the design examples, the frequency variable $\omega$ in the band of interest was uniformly digitized into $K_{\omega}=500$ evenly spaced samples. The SOCP optimization was carried out using the SeDuMi Matlab Toolbox [32] and it took less than $20 \mathrm{sec}-$ onds to obtain the solution on a Pentium $41.8 \mathrm{GHz}$ personal computer.

\section{A. Example 1: Structural PR Two-Channel Linear-Phase FIR Multi-Plet FBs}

In this example, two-channel linear-phase FIR multi-plet FBs with the following specifications are designed using the proposed approach: cutoff frequency $\omega_{c}=0.4 \pi$, passband ripples $\delta_{p 0}=\delta_{p 1}=3.45394 \times 10^{-5}$ (i.e., $3 \times 10^{-4} \mathrm{~dB}$ passband deviation) and stopband ripples $\delta_{s 0}=\delta_{s 1}=0.00316$ (i.e., $-50-\mathrm{dB}$ stopband attenuation). For these specifications, a low-order prototype FB with $\widetilde{L}_{0}=7$ and $\widetilde{L}_{1}=9$ is first designed as described in Section III-A. The specifications of this prototype FB are summarized in Table I and the corresponding frequency response is shown in Fig. 4(a). For the sake of presentation, we refer it to as prototype FB I. After applying the Euclidian algorithm, it can then be realized as the lifting structure with $L=4$ lifting steps. The corresponding lifting coefficients and scaling constants are listed in the second column of Table II. From Fig. 4(a), it can be seen that $\widetilde{\omega}_{c}$ should be chosen as $0.04 \pi$ so as to satisfy the prescribed passband and stopband ripples. In other words, we extract these passband and stopband regions of the prototype FB I and transfer their magnitudes to the transformed FB. In order for the subfilter to satisfy (2-12), its filter length $L_{Q}$ is chosen to be 16 according to (4-4). The corresponding frequency response of the transformed FB derived from prototype FB I is shown in Fig. 4(b). The specifications of the subfilter and the design results in this example are summarized in Tables III and IV, respectively. To illustrate the flexibility of the proposed transformation method, Fig. 4(c) and (d) show the frequency responses of the subfilters with different cutoff frequencies and the corresponding multi-plet FBs derived from 


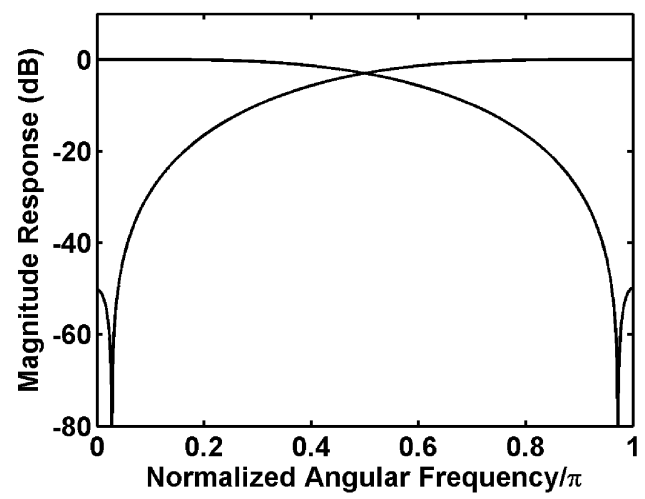

(a)

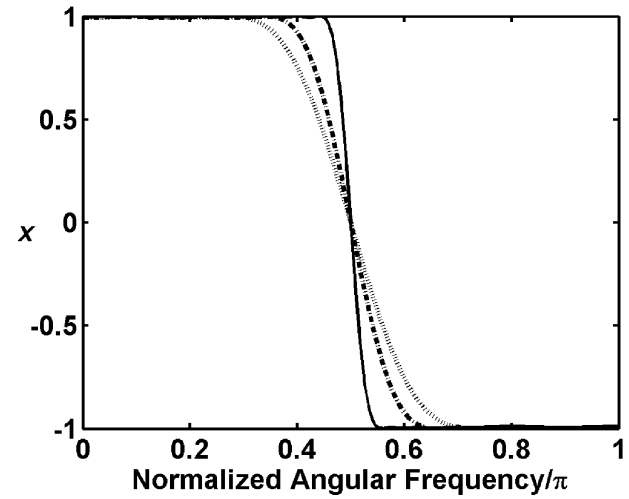

(c)

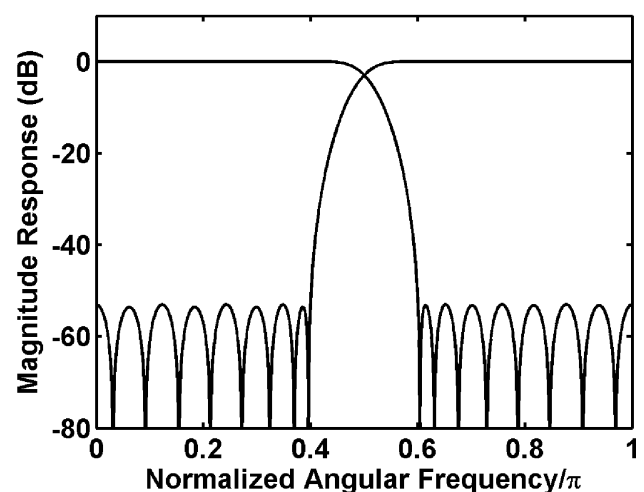

(b)

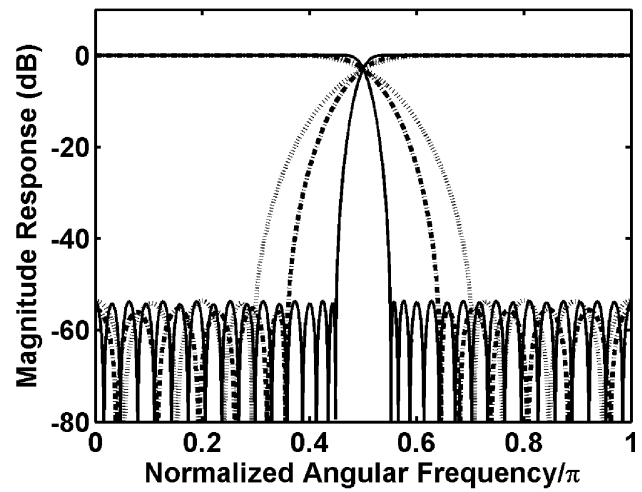

(d)

Fig. 4. Design results of linear-phase FIR multi-plet FB in Example 1. (a) Frequency responses of the analysis filter pair of prototype FB I. (b) Frequency responses of the analysis filter pair of the transformed FBs derived from prototype FB I with $\omega_{c}=0.4 \pi$. (c) and (d) Magnitude responses of the subfilters and the corresponding frequency responses of the analysis filter pairs of the transformed FBs with different cutoff frequencies (dotted line: $\omega_{c}=0.3 \pi$., dash-dotted line: $\omega_{c}=0.36 \pi$, and solid line: $\left.\omega_{c}=0.45 \pi\right)$.

TABLE I

SPECIFICATIONS AND DESIGN RESUlTS OF THE PR PROTOTYPE FBS

\begin{tabular}{c|c|c}
\hline \hline & Prototype FB I & Prototype FB II \\
\hline$\sigma$ & 0.3 & 0.3 \\
\hline$\left(\widetilde{K}_{0}, \widetilde{K}_{1}\right)$ & $(0,0)$ & $(2,2)$ \\
\hline$\widetilde{\Omega}_{c}$ & $0.1 \pi$ & $0.1 \pi$ \\
\hline Fig. & $4 \mathrm{a}$ & 5 d (dash-dotted line) \\
\hline \hline
\end{tabular}

TABLE II

LifTING COEFFICIENTS AND SCALING CONSTANTS OF THE PR PROTOTYPE FBS

\begin{tabular}{l|c|c|c}
\hline \hline & Prototype FB I & Prototype FB II & Triplet FB [8]-[11] \\
\hline$p_{0}$ & 0.23987556667257 & 0.28888910640732 & $1-\sqrt{2}$ \\
\hline$p_{1}$ & -0.54571527976115 & -0.58891192726239 & $1 / \sqrt{2}$ \\
\hline$p_{2}$ & 0.54045911345798 & 0.50121153781230 & $1-\sqrt{2}$ \\
\hline$p_{3}$ & -0.23167459250035 & -0.17093207303270 & $\mathrm{~N} / \mathrm{A}$ \\
\hline$C_{0}$ & 0.71237102180672 & 0.70252205657753 & $1 / \sqrt{2}$ \\
\hline$C_{1}$ & 0.71401331291255 & 0.69752360032606 & $1 / \sqrt{2}$ \\
\hline \hline
\end{tabular}

prototype FB I. It can be seen that the passband and stopband are equiripple and the frequency response is very symmetric. Furthermore, by realizing the subfilter as variable digital filters [33], the cutoff frequencies can be varied online. This illustrates that the proposed approach is very flexible and effective in controlling the frequency characteristics of the multi-plet FBs. As a comparison, we also consider the design of the conventional
TABLE III

SPECIFICATIONS OF THE SUBFILTERS IN EXAMPLES 1, 2 AND 3

\begin{tabular}{c|c|c|c|c}
\hline \hline & Example 1 & \multicolumn{2}{|c|}{ Example 2 } & Example 3 \\
\hline Prototype FB & $\mathrm{I}$ & $\mathrm{I}$ & $\mathrm{I}$ & $\mathrm{I}$ \\
\hline$L_{Q}$ & 16 & 32 & 36 & 32 \\
\hline$D$ & 0 & 0 & 0 & 12 \\
\hline$K_{Q}$ & 0 & $4(r=-0.9961)$ & $4(r=-0.9961)$ & $2(r=-0.9961)$ \\
\hline$\omega_{c}$ & $0.45 \pi$ & $0.45 \pi$ & $0.45 \pi$ & $0.45 \pi$ \\
\hline$\widetilde{\omega}_{c}$ & $0.04 \pi$ & $0.04 \pi$ & $0.024 \pi$ & $0.04 \pi$ \\
\hline \hline
\end{tabular}

TABLE IV

DESIGN RESUlts OF THE TRANSFORMED FBS IN EXAMPLES 1 AND 2

\begin{tabular}{c|c|c|c}
\hline \hline & Example 1 & \multicolumn{2}{|c}{ Example 2 } \\
\hline Prototype FB & $\mathrm{I}$ & $\mathrm{I}$ & $\mathrm{II}$ \\
\hline Group Delays of $H_{0}(z), H_{1}(z)$ & 45,60 & 93,124 & 105,140 \\
\hline Passband deviation of $H_{0}(z) / 10^{-3} \mathrm{~dB}$ & 0.2950 & 0.3051 & 0.4642 \\
\hline Stopband attenuation of $H_{0}(z) / \mathrm{dB}$ & 53.0565 & 53.3028 & 53.1323 \\
\hline Passband deviation of $H_{1}(z) / 10^{-3} \mathrm{~dB}$ & 0.2965 & 0.3120 & 0.4858 \\
\hline Stopband attenuation of $H_{1}(z) / \mathrm{dB}$ & 53.0219 & 53.2724 & 52.9142 \\
\hline \hline
\end{tabular}

triplet FB proposed in [10], [11]. The general design procedure of the triplet FB requires three separate designs of the subfilters and their lengths have to be chosen properly so as to satisfy different specifications. By a number of trials and errors, we 


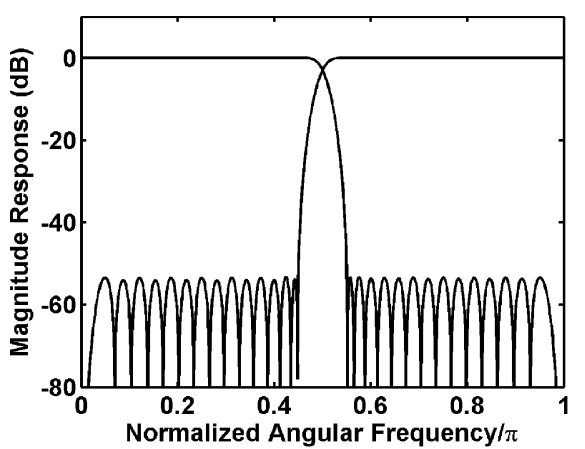

(a)

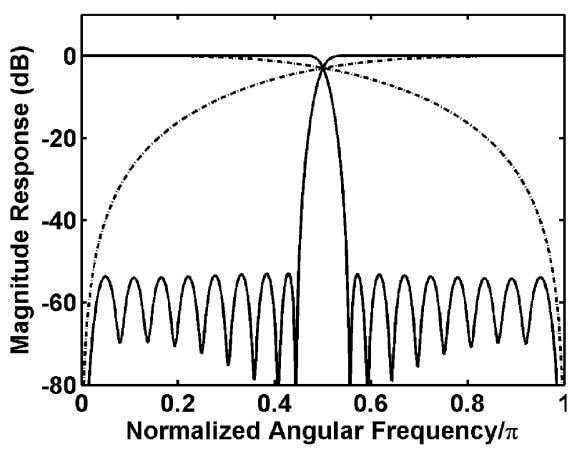

(d)

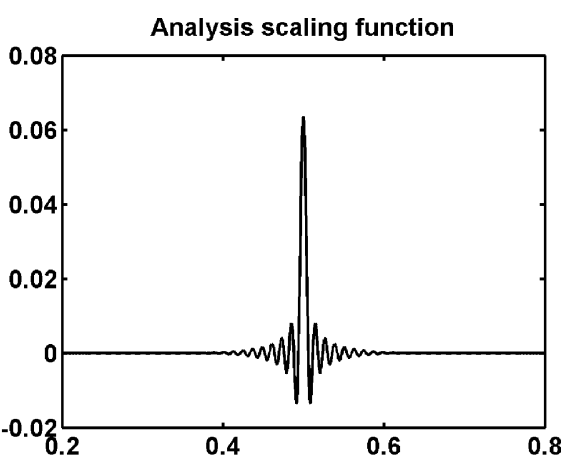

(b)

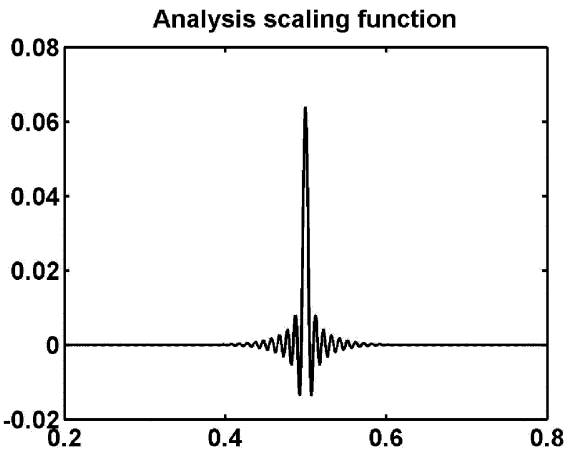

(e)

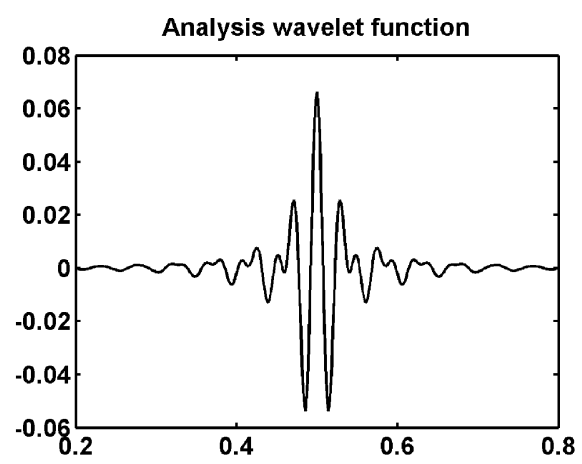

(c)

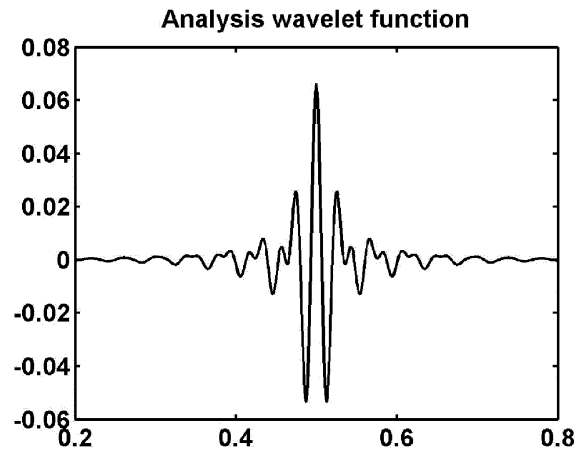

(f)

Fig. 5. Design results of linear-phase FIR multi-plet wavelet bases in Example 2 ( $K$-regularity constraint: $\left.K_{0}=K_{1}=4\right)$. (a) Frequency responses of the analysis filter pair. (b) Analysis scaling function. (c) Analysis wavelet function of the transformed FBs derived from prototype FB I with $\omega_{c}=0.45 \pi$. (d) Frequency responses of the analysis filter pair (dash-dotted line: frequency response of prototype FB II). (e) Analysis scaling function. (f) Analysis wavelet function of the transformed FBs derived from prototype FB II with $\omega_{c}=0.45 \pi$.

found that the lengths of these three subfilters have to be 22 so as to satisfy the same specifications as above with the minimum arithmetic complexity. For the proposed approach, a similar result can be obtained by designing a single subfilter of length 22 using the prototype FB in the triple structure as listed in the forth column of Table II. The results are similar and are therefore omitted for simplicity. This conventional triplet FB has comparable arithmetic complexity and performance as the abovementioned multi-plet FB. On the other hand, it has a larger difference in the system delays of the analysis filters, which would be undesirable in practical implementation. In the multi-plet FB with multiple lifting stages, a longer prototype FB can be designed to have a wider passband/stopband width (i.e., narrower transition band) so that a shorter subfilter is able to transform it to meet the same specifications. As a result, the system delays of the analysis filters can be made closer to each other. This might be useful in real time applications, where their implementations are likely to be pipelined, because less additional delay elements are required to process two subband signals. Moreover, since there is no systematic design procedure for determining the subfilter lengths, one would have to try every combination of the subfilter lengths in order to meet the specification. This is rather time consuming and makes the design inefficient. For the proposed approach, the frequency response of the prototype PR FB, such as the passband and stopband ripples, can be controlled through optimization with few variables. Identical subfilters can then be designed separately to meet the specification. In summary, the proposed multi-plet FBs employ frequency transformation of appropriate optimized prototype filters to simplify the design to a single subfilter, which reduces the delays between the two analysis filters as well as the design complexity. Moreover, the same design procedure is applicable to multiple lifting steps. Next, we shall illustrate the incorporation of prescribed $K$-regularity constraints into the transformed FBs to realize wavelet bases.

\section{B. Example 2: Structural PR Two-Channel Linear-Phase FIR Multi-Plet Wavelet Base}

In this example, two-channel linear-phase FIR multi-plet FBs with prescribed order of $K$-regularity are designed. The specifications are: $5 \times 10^{-4} \mathrm{~dB}$ passband deviation, $-50 \mathrm{~dB}$ stopband attenuation, $\omega_{c}=0.45 \pi$ and $K_{0}=K_{1}=4$. It was found that a subfilter with $\widetilde{\omega}_{c}=0.04 \pi$ and $L_{Q}=32$ is able to meet the required specifications for prototype FB I. According to Section IV-B, a prescribed order of $K$-regularity can be imposed to the multi-plet transformed FB by finding the value of $r$ in (4-15). To this end, one can verify that one of the roots of $\widetilde{R}_{0}(\widetilde{x})=0$ and $\widetilde{R}_{1}(\widetilde{x})=0$ are respectively given by $r_{0}=$ -0.9961 and $r_{1}=0.9961$. Since both of them satisfy the conditions in (4-10) and (4-13), the constraints in (4-15) with $r=$ -0.9961 can be imposed to the subfilter so that a wavelet FB can be obtained although prototype FB I is not a wavelet FB. The frequency response, analysis scaling function and analysis wavelet function of the multi-plet transformed FB so obtained with $K_{Q}=4$ are shown in Fig. 5(a)-(c), respectively. From Fig. 5(a), it can be seen that the prescribed zeros are properly imposed. This demonstrates the usefulness of the proposed SOCP method in imposing the $K$-regularity conditions.

As mentioned in Section II-C, different combinations of prototype PR FBs and subfilters can be employed to satisfy the 


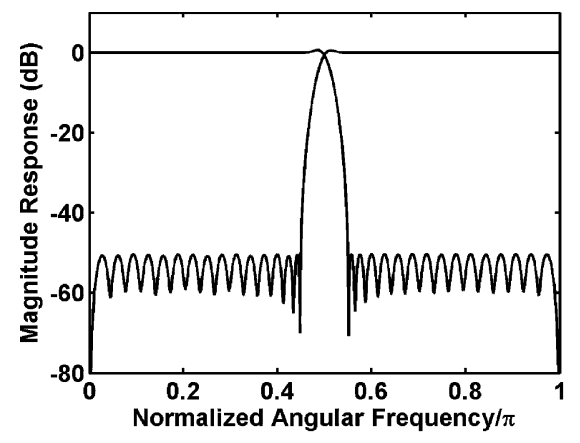

(a)

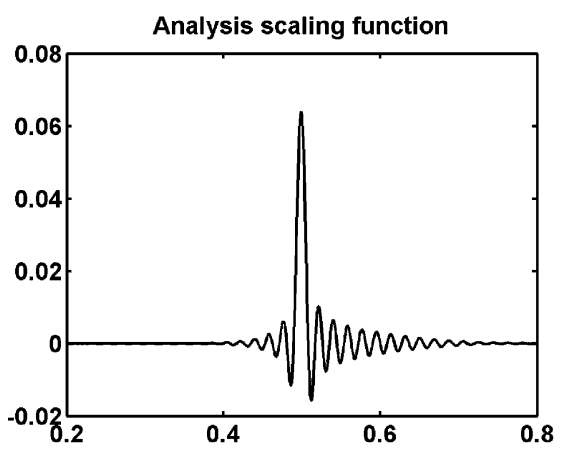

(d)

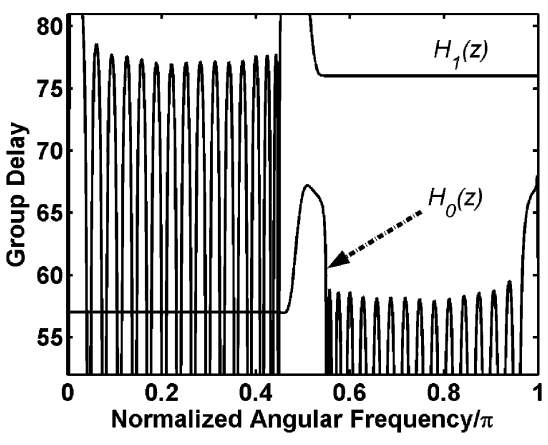

(b)

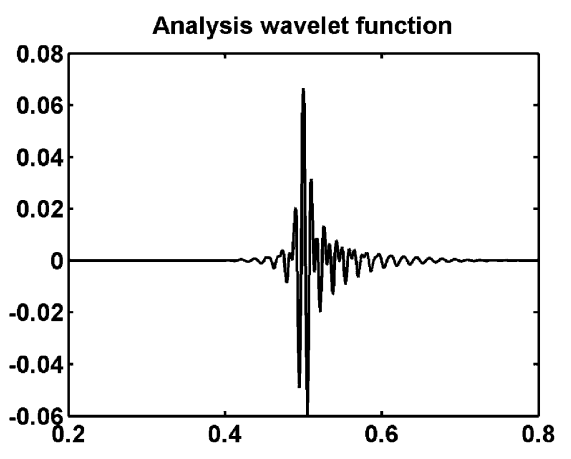

(e)

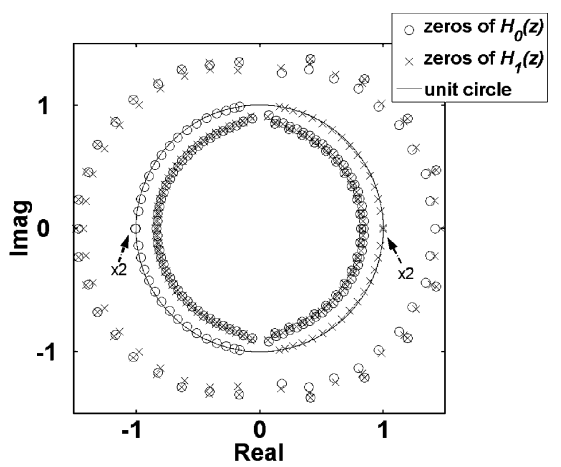

(c)

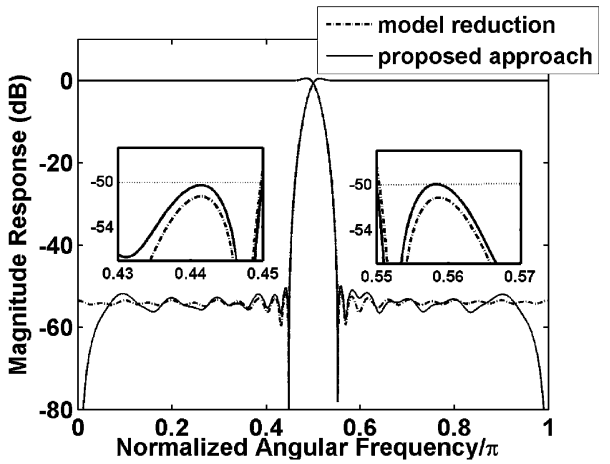

(f)

Fig. 6. Design results of low-delay FIR and IIR multi-plet wavelet bases in Example 3 ( $K$-regularity constraint: $K_{0}=K_{1}=2$ ). (a) Frequency responses of the analysis filter pair. (b) Group delay response. (c) Zeros of the analysis filters. (d) Analysis scaling function. (e) Analysis wavelet function of the low-delay FIR transformed FBs derived by the prototype FB I with $\omega_{c}=0.45 \pi$ and $D=12$. (f) Frequency responses and enlarged stopband details of the analysis filter pairs of the model-reduced FIR FB (dash-dotted line) and the proposed IIR FB (solid line).

given specifications. For illustrative purpose, another prototype FB (we call it prototype FB II for convenience) is also considered and designed to be a wavelet FB with $\widetilde{K}_{0}=\widetilde{K}_{1}=2$. The specifications, and the lifting coefficients and scaling constants of the prototype FB II are summarized in Tables I and II, respectively. The corresponding frequency response is plotted as dash-dotted line in Fig. 5(d). In order to satisfy the given specification for prototype FB II, $\widetilde{\omega}_{c}$ and $L_{Q}$ of the subfilter, as shown in the forth column of Table III, are chosen to be $0.024 \pi$ and 36 , respectively. Since longer subfilter is required, the corresponding transformed wavelet FB has slightly higher arithmetic complexity and system delay than that of the wavelet FB derived by prototype FB I. The solid line in Fig. 5(d) shows the frequency response of the wavelet FB obtained from prototype FB II with $r=-1$ and $K_{Q}=4$. The analysis scaling and wavelet functions so obtained are shown in Fig. 5(e) and (f), respectively. From the last two column of Table IV, both multi-plet transformed FBs satisfy the overall specifications although they are transformed by different prototype FBs.

\section{Example 3: Structural PR Two-Channel Low-Delay FIR and IIR Multi-Plet Wavelet Bases}

This example illustrates the flexibility of the proposed approach in designing low-delay multi-plet PR FBs and wavelets. Again, we consider the transformation of PR prototype FB I. It is shown in Table III that the specifications of the subfilter are identical to those in Example 2 except that the parameters $D$ and $K_{Q}$ of the subfilter are now set to 12 and 2, respectively. In other words, the multi-plet transformed FB is a wavelet basis and, from (2-5), the group delays of the desired analysis low-pass and high-pass filters are significantly reduced to 57 and 76 samples, as compared with 93 and 124 samples for the linear-phase case with $D=0$ in Example 2, respectively. The frequency and group delay responses of the multi-plet wavelet FB are respectively shown in Fig. 6(a) and (b). It can be seen that the low-delay multi-plet wavelet FB is approximately linear-phase in the passband with peak group delay errors of 0.0098 samples for $H_{0}(z)$, and 0.0081 samples for $H_{1}(z)$. The regularity of the analysis filter pair, $K_{0}=K_{1}=2$, was verified from the pole-zero plot of $H_{0}(z)$ and $H_{1}(z)$ in Fig. 6(c). The analysis scaling and wavelet functions are, respectively, shown in Fig. 6(d)-(e). They are rather smooth, but not symmetric due to the low-delay constraint. The performances of the analysis filter pair are summarized in Table V. It can be seen that the passband and stopband ripple errors are slightly increased, as compared with its linear-phase counterpart in Example 2, in exchange for a much lower system delay.

Alternatively, IIR subfilters can be employed to further lower the arithmetic complexity. As discussed in Section V, the model reduction technique in [18] and [19] is applied to obtain the desired IIR subfilters from the designed FIR subfilter above. With $L_{G, m}=L_{D}=13, m=0, \ldots, L-1$, the number of multipliers and adders are respectively 100 and 96 , which are about $78 \%$ of those required for the FIR case. However, it can be seen from the dash-dotted lines in Fig. 6(f) that the LS solution exhibits significant ripples near the band edges and the stopband attenuation is significantly worsen. To improve the perfomance, peak stopband constraints of $50 \mathrm{~dB}$ and $K$-regularity constraints are 
TABLE V

DESIGN Results of THE TRANSFORMED FBS DERIVED From PROTOTYPE FB I IN EXAMPLE 3

\begin{tabular}{c|c|c|c}
\hline \hline & $\begin{array}{c}\text { Low-delay } \\
\text { FIR }\end{array}$ & {$[30]$} & $\begin{array}{c}\text { Low-delay } \\
\text { IIR }\end{array}$ \\
\hline Group Delays of $H_{0}(z), H_{1}(z)$ & 57,76 & 57,76 & 57,76 \\
\hline Group delay error of $H_{0}(z) /$ samples & 0.0098 & 0.0096 & 0.0126 \\
\hline Passband deviation of $H_{0}(z) / 10^{-3} \mathrm{~dB}$ & 0.3964 & 0.28 & 0.3629 \\
\hline Stopband attenuation of $H_{0}(z) / \mathrm{dB}$ & 50.34 & 48.08 & 50 \\
\hline Group delay error of $H_{1}(z) / \mathrm{samples}$ & 0.0081 & 0.0078 & 0.0111 \\
\hline Passband deviation of $H_{1}(z) / 10^{-3} \mathrm{~dB}$ & 0.3916 & 0.28 & 0.3881 \\
\hline Stopband attenuation of $H_{1}(z) / \mathrm{dB}$ & 50.42 & 49.08 & 50 \\
\hline Number of multipliers & 128 & 100 & 100 \\
\hline Number of adders & 124 & 96 & 96 \\
\hline \hline
\end{tabular}

imposed during the model reduction of the above FIR subfilter. From the solid line in Fig. 6(f), it can be seen that the maximum stopband attenuation of the proposed IIR FB is now increased from $48.08 \mathrm{~dB}$ and $49.08 \mathrm{~dB}$ to $50 \mathrm{~dB}$ for $H_{0}\left(e^{j \omega}\right)$ and $H_{1}\left(e^{j \omega}\right)$, respectively. Also, as depicted in Fig. 6(f) and the pole zero plot (not shown here due to page limitation), $H_{0}(z)$ and $H_{1}(z)$ have two zeros at $\omega=\pi$ and $\omega=0$, respectively. The design results in this example are summarized in Table V. Finally, it should be noted that the multi-plet FB can be efficiently implemented using sum-of-power-of-two (SOPOT) coefficient and the multiplier block technique. This gives rise to very efficient multiplier-less realization. Interested readers are referred to [4] for more information in the multiplier-less realization of the structural PR FB in [1]. Furthermore, like the triplet FBs in [11], the proposed multi-plet FB can be cascaded to form $M$-channel PR FBs where $M$ is an integer power of two.

\section{CONCLUSION}

A new class of two-channel structural PR FIR/IIR FBs called multi-plet FBs is presented. It generalizes the structural PR FBs and triplet FBs by employing multiple lifting steps as in conventional lifting structure. The design of the multi-plet FBs can be done in two separate steps: i) a low-order prototype PR FB with a much wider transition band is first designed using nonlinear optimization in order to obtain a symmetric frequency response and prescribed passband/stopband ripples; and ii) a subfilter is then designed using SOCP so that the prototype FB can be warped by means of frequency transformation to meet the desired transition bandwidth, while preserving the PR condition, passband/stopband ripples and the lifting structure. Under the SOCP framework, linear equalities such as the $K$-regularity constraints can be easily incorporated in the design of the subfilter to obtain muti-plet-based wavelet bases. To further reduce the system delay of the proposed multi-plet FBs, the design of low-delay FIR and causal stable IIR subfilters is also considered. Design examples show that the proposed approaches provide more flexibility in controlling the frequency characteristic of the PR FBs and lower design complexity than conventional methods.

\section{REFERENCES}

[1] S. M. Phoong, C. W. Kim, P. P. Vaidyanathan, and R. Ansari, "A new class of two-channel biothogonal filter banks and wavelet bases," IEEE Trans. Signal Process., vol. 43, no. 3, pp. 649-664, Mar. 1995.

[2] J. S. Mao, S. C. Chan, W. Liu, and K. L. Ho, "Design and multiplierless implementation of a class of two-channel PR FIR filterbanks and wavelets with low system delay," IEEE Trans. Signal Process., vol. 48, no. 12, pp. 3379-3394, Dec. 2000.

[3] D. B. H. Tay, "Two stage, least squares design of biorthogonal filter banks," in Proc. IEEE ISCAS'2000, Sep. 2000, vol. 1, pp. 591-594.

[4] S. C. Chan, C. K. S. Pun, and K. L. Ho, "New design and realization techniques for a class of perfect reconstruction two-channel FIR filterbanks and wavelets bases," IEEE Trans. Signal Process., vol. 52, no. 7 , pp. 2135-2141, Jul. 2004.

[5] X. Zhang and T. Yoshikawa, "Design of causal IIR perfect reconstruction filter banks," in Proc. IEEE ICASSP'1998, May 1998, vol. 3, pp. $1429-1432$.

[6] D. B. H. Tay, "Design of causal stable IIR perfect reconstruction filter banks using transformation of variables," in Proc. IEEE ISCAS'1997, Jun. 1997, vol. 3, pp. 2425-2428.

[7] R. Ansari, C. W. Kim, and M. Dedovic, "Structure and design of twochannel filter banks derived from a triplet of halfband filters," IEEE Trans. Circuits Syst. II, Analog Digit. Signal Process., vol. 46, no. 12, pp. 1487-1496, Dec. 1999.

[8] D. B. H. Tay, "Least squares design of the class of triplet halfband filter banks," in Proc. IEEE ISCAS'2001, 2001, vol. 2, pp. 481-484.

[9] _ - "A novel approach to the design of the class of triplet halfband filterbanks," IEEE Trans. Circuits Syst. II, Exp. Briefs, vol. 57, no. 7, pp. 378-383, Jul. 2004.

[10] K. S. Yeung and S. C. Chan, "The design of two-channel perfect reconstruction FIR triplet wavelet filter banks using semidefinite programming," in Proc. IEEE ISCAS'2003, May 2003, vol. 4, pp. 253-256.

[11] S. C. Chan and K. S. Yeung, "On the design and multiplier-less realization of perfect reconstruction triplet-based FIR filter banks and wavelet bases," IEEE Trans. Circuits Syst. I, Reg. Papers, vol. 51, no. 8, pp. 1476-1791, Aug. 2004.

[12] W. Sweldens, "The lifting scheme: A new philosophy in biorthogonal wavelet construction," in Proc. SPIE Wavelet Appl. Signal Image Process. III, Jul. 1995, vol. 2569, pp. 68-79.

[13] I. Daubechies and W. Sweldens, "Factoring wavelet transform into lifting steps," J. Fourier Anal. Appl., vol. 4, no. 3, pp. 247-269, 1998.

[14] T. Saramaki, "Design of FIR filters as a tapped cascaded interconnection of identical subfilters," IEEE Trans. Circuits Syst., vol. 34, pp. 1011-1029, Sep. 1987.

[15] J. F. Kaiser and R. W. Hamming, "Sharpening the response of a symmetric nonrecursive filter by multiple use of the same filter," IEEE Trans. Acoust. Speech, Signal Process., vol. ASSP-25, no. 10, pp. 415-422, Oct. 1977.

[16] T. G. Marshall Jr., "Zero-phase filter bank and wavelet coder matrices: Properties, triangular decompositions, and a fast algorithm," Multidim. Syst. Signal Process., vol. 7, no. 3/4, pp. 71, 88, 1996.

[17] R. E. Van Dyck, T. G. Marshall Jr., M. Chin, and N. Moayeri, "Wavelet video coding with ladder structures and entropy-constrained quantization," IEEE Trans. Circuits Syst. I, Fundam. Theory Appl., vol. 6, no. 10, pp. 483-495, Oct. 1996.

[18] S. C. Chan, K. M. Tsui, and K. W. Tse, "Design of constrained IIR and interpolated IIR filters using a new semi-definite programming based model reduction technique," in Proc. EUSIPCO'2004, Sep. 6-10, 2004, pp. 1245-1248.

[19] — "Design of constrained causal stable IIR filters using a new second-order cone programming based model reduction technique," IEEE Trans. Circuits Systs. II, to be published.

[20] D. B. H. Tay and N. G. Kingsbury, "Flexible design of multidimensional perfect reconstruction FIR 2-band filters using transformations of variables," IEEE Trans. Image Process., vol. 2, no. 10, pp. 466-480, Oct. 1993.

[21] K. M. Tsui and S. C. Chan, "On the design of two-channel 2-D nonseparable multi-plet perfect reconstruction filter banks," in Proc. IEEE ISCAS'2006, May 2006, pp. 2033-2036.

[22] M. S. Lobo, L. Vandenberghe, S. Boyd, and H. Lebret, "Applications of second-order cone programming," Linear Algebra Appl., vol. 248, pp. 193-228, Nov. 1998

[23] W. S. Lu and T. Hinamoto, "Optimal design of FIR frequencyresponse-masking filters using second-order cone programming," in Proc. IEEE ISCAS'2003, May 2003, vol. 3, pp. 878-881. 
[24] J. O. Coleman and D. P. Scholnik, "Design of nonlinear phase FIR filters with second-order cone programming," in Proc. IEEE MWSCAS 1999, Aug. 1999, vol. 1, pp. 409-412.

[25] K. M. Tsui, S. C. Chan, and K. S. Yeung, "Design of FIR digital filters with prescribed flatness and peak error constraints using secondorder cone programming," IEEE Trans. Circuits Syst. II, vol. 52, pp. 601-605, Sep. 2005.

[26] H. Wolkowicz, R. Saigal, and L. Vandenberge, Handbook of Semidefinite Programming: Theory, Algorithms, and Applications. Norwell, MA: Kluwer, 2000.

[27] D. B. H. Tay, "Design of perfect reconstruction filter banks using transformation of variables and re-factorization," in Proc. ICICS'1997, Sep. 1997, vol. 3, pp. 1349-1352.

[28] — , "Parametric bernstein polynomial for least squares design of 3-D wavelet filter banks," IEEE Trans. Circuits Syst. I, Fundam. Theory Appl., vol. 49, no. 6, pp. 887-891, Jun. 2002.

[29] P. P. Vaidyanathan, Multirate Systems and Filter Banks. Englewood Cliffs, NJ: Prentice-Hall, 1993.

[30] A. V. Oppenheim, R. W. Schafer, and J. R. Buck, Discrete-Time Signal Processing. Upper Saddle River, NJ: Prentice-Hall, 1999.

[31] H. Brandenstein and R. Unbehauen, "Least-squares approximation of FIR by IIR filters," IEEE Trans. Signal Process., vol. 46, no. 1, pp. 21-30, Jan. 1998.

[32] J. F. Sturm, "Using SeDuMi 1.02, a MATLAB toolbox for optimization over symmetric cones," Optim. Math. Softw., vol. 11-12, pp. 625-653, 1999.

[33] K. S. Pun, S. C. Chan, K. S. Yeung, and K. L. Ho, "On the design and implementation of FIR and IIR digital filters with variable frequency characteristics," IEEE Trans. Circuits Syst. II, Analog Digit. Signal Process., vol. 49, no. 11, pp. 689-703, Nov. 2002.

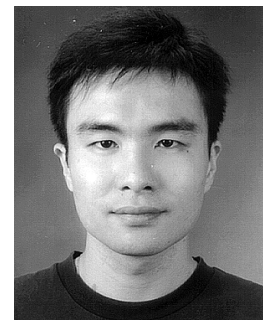

K. M. Tsui received the B.Eng. and M.Phil. degrees in electrical and electronic engineering from The University of Hong Kong, Hong Kong, in 2001 and 2004 , respectively. He is currently working toward the Ph.D. degree in the Department of Electrical and Electronic Engineering at the same university.

His main research interests are in biomedical signal processing, digital signal processing, multirate filter bank and wavelet design, and digital filter design: realization and application.

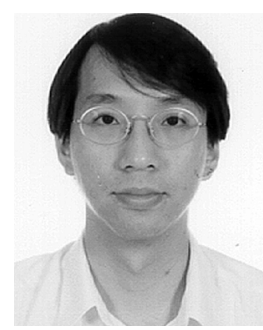

S. C. Chan (S'87-M'92) received the B.Sc. (Eng.) and Ph.D. degrees from the University of Hong Kong, Hong Kong, in 1986 and 1992, respectively.

He joined City Polytechnic of Hong Kong, Hong Kong in 1990 as an Assistant Lecturer and later as a University Lecturer. Since 1994, he has been with the Department of Electrical and Electronic Engineering, the University of Hong Kong, and is now an Associate Professor. He was a Visiting Researcher in Microsoft Corporation, Redmond, CA and Microsoft China in 1998 and 1999, respectively. His research interests include fast transform algorithms, filter design and realization, multirate signal processing, communications signal processing, and image-based rendering.

Dr. Chan is currently a member of the Digital Signal Processing Technical Committee of the IEEE Circuits and Systems Society. He was Chairman of the IEEE Hong Kong Chapter of Signal Processing from 2000 to 2002. 